\title{
Hamiltonian stability of Hamiltonian minimal Lagrangian submanifolds in pseudo- and para-Kähler manifolds
}

\author{
Henri Anciaux, Nikos Georgiou ${ }^{\dagger}$
}

November 8, 2018

\begin{abstract}
Let $\mathcal{L}$ be a Lagrangian submanifold of a pseudo- or para-Kähler manifold which is H-minimal, i.e. a critical point of the volume functional restricted to Hamiltonian variations. We derive the second variation of the volume of $\mathcal{L}$ with respect to Hamiltonian variations. We apply this formula to several cases. In particular we observe that a minimal Lagrangian submanifold $\mathcal{L}$ in a Ricci-flat pseudo- or para-Kähler manifold is $\mathrm{H}$-stable, i.e. its second variation is definite and $\mathcal{L}$ is in particular a local extremizer of the volume with respect to Hamiltonian variations. We also give a stability criterion for spacelike minimal Lagrangian submanifolds in para-Kähler manifolds, similar to Oh's stability criterion for minimal Lagrangian manifolds in Kähler-Einstein manifolds (cf [Oh1]). Finally, we determine the H-stability of a series of examples of H-minimal Lagrangian submanifolds: the product $\mathbb{S}^{1}\left(r_{1}\right) \times \ldots \times \mathbb{S}^{1}\left(r_{n}\right)$ of $n$ circles of arbitrary radii in complex space $\mathbb{C}^{n}$ is $\mathrm{H}$-unstable with respect to any indefinite flat Hermitian metric, while the product $\mathbb{H}^{1}\left(r_{1}\right) \times \ldots \times \mathbb{H}^{1}\left(r_{n}\right)$ of $n$ hyperbolas in para-complex vector space $\mathbb{D}^{n}$ is H-stable for $n=1,2$ and $\mathrm{H}$ unstable for $n \geq 3$. Recently, minimal Lagrangian surfaces in the space of geodesics of space forms have been characterized ([An3], see also [Ge]); on the other hand, a class of H-minimal Lagrangian surfaces in the tangent bundle of a Riemannian, oriented surface has been identified in [AGR]. We discuss the H-stability of all these examples.
\end{abstract}

2000 MSC: 53D12, 49Q05, 53A07

\section{Introduction}

A submanifold of a pseudo-Riemannian manifold $(\mathcal{M}, g)$ is said to be minimal if it is a critical point of the volume functional associated to $g$. The first variation formula characterizes a minimal submanifold by the vanishing of the trace of its second fundamental form, the mean curvature vector. Minimality is therefore the first order condition for a submanifold to be volume-extremizing in its homology class. In [Si], the second order condition to be satisfied by a volume-extremizing submanifold has been derived in the Riemannian case (see [An2] for the generalization in the pseudo-Riemannian setting). Those minimal submanifolds satisfying this condition (hence local extremizers of the volume) are called stable minimal submanifolds.

*Universidade de São Paulo; supported by CNPq (PQ 302584/2007-2) and Fapesp (2010/18752-0)

$\dagger$ Department of Mathematics and Statistics, University of Cyprus; partially supported by Fapesp (2010/08669-9) 
When the ambient manifold $\mathcal{M}$ is in addition symplectic, it is interesting to look at those submanifolds on which the symplectic form vanish, namely, Lagrangian submanifolds. It was discovered in [HL1] that minimal Lagrangian submanifolds of a Ricci-flat Kähler manifold (usually called Calabi-Yau manifolds) have the striking property of being calibrated. A classical argument using Stokes theorem shows that calibrated submanifolds are volume-extremizing, and therefore automatically stable. On the other hand, since the Lagrangian class is closed under the action of Hamiltonian diffeomorphisms, it is natural to pose the problem of extremizing the volume of a Lagrangian submanifold in its Hamiltonian isotopy class. A Lagrangian submanifold is said to be H-minimal (or H-stationary) if is a critical point of the volume functional with respect to such Hamiltonian variations. A H-minimal Lagrangian submanifold is characterized by the fact that its mean curvature vector is divergence-free. A H-minimal Lagrangian submanifold will be said to be $H$-stable if the second variation of the volume functional, with respect to Hamiltonian variations again, is a definite quadratic form.

In [Oh1] and [Oh2], the second variation formula of a H-minimal Lagrangian submanifold has been derived in the case of a Kähler manifold. In the special case in which $(i)$ the submanifold $\mathcal{L}$ is not only $\mathrm{H}$-minimal but minimal and $(i i)$ the ambient manifold $\mathcal{M}$ is Kähler-Einstein, the second variation operator depends only on the induced metric on $\mathcal{L}$ and it is easy to prove that the $\mathcal{L}$ is $\mathrm{H}$-stable if and only if the first eigenvalue of the Laplacian of the induced metric on $\mathcal{L}$ is larger than the scalar curvature of $\mathcal{M}$ divided by its dimension ([Oh1]). This stability criterion allowed several classification results: for example, the Clifford torus is the unique H-stable minimal Lagrangian torus in complex projective plane $\mathbb{C P}^{2}$ ([Ha],[Ur1]), and the unique $\mathrm{H}$-stable compact minimal orientable Lagrangian surface of $\mathbb{C P}^{2}$ with genus less or equal to 4 ([Ur2]). There are also some results about minimal Lagrangian submanifolds in the space $L\left(\mathbb{S}^{n}\right)$ of geodesics of the unit sphere $\mathbb{S}^{n}([\mathrm{~Pa}])$. On the other hand, it follows from the second variation formula that the product of $r_{1} \mathbb{S}^{1} \times \ldots \times r_{n} \mathbb{S}^{1}$ of $n$ circles of arbitrary radii in complex Euclidean space $\mathbb{C}^{n}$, which is H-minimal but not minimal, is $\mathrm{H}$-stable ([Oh2]). This leads to the so-called Oh's conjecture, which states that the product of $r_{1} \mathbb{S}^{1} \times \ldots \times r_{n} \mathbb{S}^{1}$ is a global minimizer of the volume in its Hamiltonian isotopy class. In spite of some partial answers ([An1]), the question is still open.

There are two natural extensions of Kähler geometry to the pseudo-Riemannian setting: a pseudoKähler structure $(J, g)$ on a manifold $\mathcal{M}$ is defined by the same axioms than a classical Kähler one, dropping the requirement that the metric $g$ is definite. The fact that $J$ is an isometry implies that the signature of $g$ must be even. Moreover, if the signature of $g$ is $(2 p, 2(n-p))$, the signature of the induced metric on a Lagrangian submanifold, if non degenerate, must be $(p, n-p)$. On the other hand, a para-Kähler structure is a pair $(J, g)$, with the same properties than a Kähler one, except that $J$ is para-complex rather than complex, i.e. we have $J^{2}=I d$, and that the compatibility of $g$ with respect to $J$ becomes $g(J ., J)=.-g(.,$.$) . The latter equation implies that the signature$ of $g$ is neutral, but places no restriction on the induced metric of a non-degenerate Lagrangian submanifold. The simplest example of para-Kähler manifold is the Cartesian $n$-product $\mathbb{D}^{n}$ of the set of para-complex (or split-complex, or double) numbers $\mathbb{D}$, (see Section 1 for a precise definition of pseudo- and para-Kähler structures, as well as a description of $\mathbb{D}^{n}$ ).

Recently there have been growing interest about submanifold theory in the pseudo-Riemannian setting. In [Me] (cf also [HL2]), the concept of calibration is extended to the realm of pseudoRiemannian geometry. In [Dg] (cf also [An3]), the study of minimal Lagrangian submanifolds in complex space $\mathbb{C}^{n}$ endowed with a flat pseudo-Hermitian form is addressed. It also has been 
observed that the spaces of geodesics of space forms enjoy natural pseudo- or para-Kähler structures (cf [GG1], [AGK], [An4]).

The purpose of this paper is the study of the Hamiltonian stability of H-minimal Lagrangian submanifolds in pseudo- and para-Kähler manifolds. In particular, we calculate the second variation of volume with respect to Hamiltonian variations:

Main Theorem: Let $(\mathcal{M}, J, g)$ be a pseudo- or para-Kähler $2 n$-dimensional manifold with Ricci curvature tensor Ric. Let $\mathcal{L}$ be a H-minimal Lagrangian submanifold of $\mathcal{M}$ with mean curvature vector $\vec{H}$ and second fundamental form $h$. Denote respectively by $\Delta$ and $\nabla$ the Laplacian and the gradient of the induced metric on $\mathcal{L}$. Then, given $u \in C_{c}^{\infty}(\mathcal{L})$ a smooth, compactly supported real function on $\mathcal{L}$ and $X=J \nabla u$ the corresponding Hamiltonian vector field, the second variation formula $\delta^{2} \mathcal{V}(\mathcal{L})(X)$ of the volume of $\mathcal{L}$ with respect to $X$ is given by:

$$
\delta^{2} \mathcal{V}(\mathcal{L})(X)=\int_{\mathcal{L}} \epsilon\left((\Delta u)^{2}-\operatorname{Ric}(\nabla u, \nabla u)-2 g(n \vec{H}, h(\nabla u, \nabla u))\right)+g(n \vec{H}, J \nabla u)^{2},
$$

where $\epsilon=1$ in the Kähler case and $\epsilon=-1$ in the para-Kähler one (here and in the following, all the integrals are meant with respect to the volume element induced by the metric).

We observe that our notation differs from that of [Oh2], since we describe Hamiltonian variations via test functions, instead of using exact 1-forms as in [Oh2]. Of course both viewpoints are equivalent and so in the Kähler case we recover Oh's main result. A first, immediate consequence of this formula is obtained when the last three terms of this formula vanish:

Corollary 1 Let $(\mathcal{M}, J, g)$ be a Ricci-flat pseudo-or para-Kähler manifold and let $\mathcal{L}$ be a minimal Lagrangian submanifold of $\mathcal{M}$. Then $\delta^{2} \mathcal{V}(\mathcal{L})(X)=\epsilon \int_{\mathcal{L}}(\Delta u)^{2}$, which implies:

- in the pseudo-Kähler case, $\mathcal{L}$ is a local minimizer of the volume in its Hamiltonian isotopy class;

- in the para-Kähler case, $\mathcal{L}$ is a local maximizer of the volume in its Hamiltonian isotopy class.

It has been proved in [Me] (see also [HL2]) in the para-Kähler case, and in [An3] in the pseudoKähler case, that minimal Lagrangian submanifolds of a Ricci-flat manifold enjoy a kind of "Lagrangian calibration" and are therefore extremizers of the volume in their Lagrangian isotopy class. Hence Corollary 1 may be regarded as the local version of the results of [Me] and [An3].

We also get a result similar to Oh's Stability criterion for compact, minimal Lagrangian submanifolds with definite induced metric in a para-Kähler Einstein manifold:

Theorem 1 Let $(\mathcal{M}, J, g)$ be a para-Kähler Einstein manifold of dimension $2 n$ with scalar curvature $2 n c$ and $\mathcal{L}$ be a compact, minimal, Lagrangian submanifold of $\mathcal{M}$ whose induced metric is definite. Then $\mathcal{L}$ is a local maximizer of the volume in its Hamiltonian isotopy class if and only if the first eigenvalue of the Laplacian of the induced metric on $\mathcal{L}$ satisfies $\lambda_{1} \geq c$.

Next, we turn our attention to two natural examples of H-minimal Lagrangian submanifolds which are not minimal. It turns out that the homogeneous tori of complex space $\mathbb{C}^{n}$, though still Hminimal when the ambient metric is indefinite, are no longer H-stable. Hence there is no counterpart of Oh's conjecture in the indefinite case: 
Theorem 2 Let $\mathbb{T}_{r_{1}, \ldots, r_{n}}^{n}:=\mathbb{S}^{1}\left(r_{1}\right) \times \ldots \times \mathbb{S}^{1}\left(r_{n}\right)$ be the product of $n$ circles of arbitrary radii $r_{1}, \ldots, r_{n}$ in complex space $\mathbb{C}^{n}$ equipped with the pseudo-Kähler structure given by the Hermitian form

$$
\langle\langle., .\rangle\rangle_{p}:=-\sum_{j=1}^{p} d z_{j} d \bar{z}_{j}+\sum_{j=p+1}^{n} d z_{j} d \bar{z}_{j} .
$$

Then $\mathbb{T}_{r_{1}, \ldots, r_{n}}^{n}$ is Lagrangian and H-minimal. Moreover, if $p \neq 0, n$, it is H-unstable.

It seems reasonable to expect that Hamiltonian instability is shared by all non-minimal Lagrangian submanifolds, at least in the flat case:

Conjecture 1 Let $\mathcal{L}$ be a H-minimal, non minimal, Lagrangian submanifold in complex space $\mathbb{C}^{n}$ equipped with the pseudo-Kähler structure given by the Hermitian form $\langle\langle., .\rangle\rangle_{p}$, with $p \neq 0, n$. Then $\mathcal{L}$ is H-unstable.

The situation of the para-Kähler equivalent of the tori $\mathbb{T}_{r_{1}, \ldots, r_{n}}^{n}$, namely the product of hyperbolas in $\mathbb{D}^{n}$, is different and somewhat surprising since here the $\mathrm{H}$-stability depend on their dimension, and not on their induced metric:

Theorem 3 Denote by $\mathbb{H}_{+}^{1}(r):=\left\{(x, y) \in \mathbb{D} \mid x^{2}-y^{2}=r^{2}, x>0\right\}$ and $\mathbb{H}_{-}^{1}(r):=\{(x, y) \in$ $\left.\mathbb{D} \mid x^{2}-y^{2}=-r^{2}, y>0\right\}$ the hyperbolas of "radius" $r$ of the set of para-complex numbers. Let $\mathbb{H}_{r_{1}, \ldots, r_{n}}^{n}:=\mathbb{H}_{ \pm}^{1}\left(r_{1}\right) \times \ldots \times \mathbb{H}_{ \pm}^{1}\left(r_{n}\right)$ the product of $n$ hyperbolas of arbitrary signature and radii $r_{1}, \ldots, r_{n}$ in para-complex space $\mathbb{D}^{n}$ equipped with its canonical para-Kähler structure (see Section 1). Then $\mathbb{H}_{r_{1}, \ldots, r_{n}}^{n}$ is Lagrangian and H-minimal. Morever, the curves $\mathbb{H}_{ \pm}^{1}(r)$ and the surfaces $\mathbb{H}_{r_{1}, r_{2}}^{2}$ are $H$-stable, while $\mathbb{H}_{r_{1}, \ldots, r_{n}}^{n}, n \geq 3$, is H-unstable.

Finally, we compute the stability of some Lagrangian surfaces in two examples of non-flat pseudoor para-Kähler manifolds. First, it has been recently discovered ([Sa],[GG1],[AGK]) that the spaces of $L^{+}\left(\mathbb{S}_{p}^{3}\right)$ and $L^{-}\left(\mathbb{S}_{p}^{3}\right)$ positive and negative geodesics of 3-dimensional space form $\mathbb{S}_{p}^{3}$ enjoy two natural pseudo- or para-Kähler structure $(\mathbb{J}, \mathbb{G})$ and $\left(\mathbb{J}^{\prime}, \mathbb{G}^{\prime}\right)$ which share the same symplectic form $\omega=\epsilon \mathbb{G}(\mathbb{J} .,)=.\epsilon^{\prime} \mathbb{G}^{\prime}\left(\mathbb{J}^{\prime} .,.\right)$. It has been proved in $[\mathrm{An} 4]$ that $\mathbb{G}$ is Einstein and that $\mathbb{G}^{\prime}$ is scalar flat, and that Lagrangian surfaces of $L^{ \pm}\left(\mathbb{S}_{p}^{3}\right)$ arise as normal congruences (or Gauss maps) of surfaces of $\mathbb{S}_{p}^{3}$. Moreover, the normal congruence of a tube over a geodesic of $\mathbb{S}_{p}^{3}$ is minimal with respect to both metrics $\mathbb{G}$ and $\mathbb{G}^{\prime}$. The next result discusses the H-stability of such surfaces:

Theorem 4 Let $\overline{\mathcal{S}} \subset L^{ \pm}\left(\mathbb{S}_{p}^{3}\right)$ be the normal congruence of a the tube over a geodesic $\gamma$ of $\mathbb{S}_{p}^{3}$. Then:

- $\overline{\mathcal{S}}$ is H-stable with respect to $\mathbb{G}$ if and only if $p=2$, i.e. $\mathbb{S}_{2}^{3}$ is the anti-de Sitter space and $\gamma$ is an unbounded geodesic;

- $\overline{\mathcal{S}}$ is $H$-stable with respect to $\mathbb{G}^{\prime}$ if and only if $\gamma$ is a closed geodesic (this implies that $p=0$ or 2 , i.e. $\mathbb{S}_{p}^{3}$ is the Riemannian sphere or the anti-de Sitter space).

The last situation we study it the case of the tangent bundle $T \mathcal{N}$ of a oriented, Riemannian surface $\left(\mathcal{N}, g_{0}\right)$. In $[\mathrm{GK}]$, it has been proved that $T \mathcal{N}$ enjoys a natural pseudo-Kähler structure $(\mathbb{J}, \mathbb{G})$. Moreover, it is observed in $[\mathrm{AGR}]$ that given a regular curve $\gamma$ of $\mathcal{N}$, the set of tangent vectors to $\mathcal{N}$ which are normal to $\gamma$ (thus a surface in $T \mathcal{N}$ ) is Lagrangian and H-minimal (these surfaces are called normal bundles in $[\mathrm{AGR}])$. 
Theorem 5 Let $\left(\mathcal{N}, g_{0}\right)$ be a Riemannian surface with Gaussian curvature $K, \gamma$ a regular curve of $\mathcal{N}$ with geodesic curvature $\kappa$ and $\mathcal{L}$ its normal bundle.

- If $\kappa^{2} \leq-2 K$ along $\gamma$ (this is for example the case if $\gamma$ is a geodesic of a surface $\mathcal{N}$ with negative curvature), then $\mathcal{L}$ is $\mathrm{H}$-stable;

- If $\gamma$ is closed and $\sup _{\gamma}\left(\kappa^{2}+2 K\right) \leq \frac{16 \pi^{2}}{L^{2}}$, where $L$ is the length of $\gamma$, then $\mathcal{L}$ is $H$-stable;

- If $\gamma$ is unbounded and $\kappa^{2}>-2 K$ along $\gamma$ (this is for example the case any curve of a surface with non-negative curvature), then $\mathcal{L}$ is $H$-unstable.

Acknowledgements. The authors would like to thank G. Siciliano for his helpful comments about the proof of Theorems 3 and 5 .

\section{Preliminaries}

\subsection{The space $\mathbb{D}^{n}$}

The set of para-complex (or split-complex, or double) numbers is the two-dimensional real vector space $\mathbb{R}^{2}$ endowed with the commutative algebra structure whose product rule is given by

$$
(x, y) \cdot\left(x^{\prime}, y^{\prime}\right)=\left(x x^{\prime}+y y^{\prime}, x y^{\prime}+x^{\prime} y\right) .
$$

The number $(0,1)$, whose square is $(1,0)$ and not $(-1,0)$, will be denoted by $\tau$. It is convenient to use the following notation: $(x, y) \simeq z=x+\tau y$. In particular, one has the same conjugation operator than in $\mathbb{C}$ :

$$
\overline{x+\tau y}=x-\tau y,
$$

whose corresponding square norm is

$$
|z|^{2}:=z \cdot \bar{z}=x^{2}-y^{2} .
$$

In other words, the metric associated to $|.|^{2}$ is the Minkowski metric $d x^{2}-d y^{2}$.

We define the "para-Cauchy-Riemann" operators on $\mathbb{D}$ by

$$
\frac{\partial}{\partial z}:=\frac{1}{2}\left(\frac{\partial}{\partial x}+\tau \frac{\partial}{\partial y}\right) \quad \frac{\partial}{\partial \bar{z}}:=\frac{1}{2}\left(\frac{\partial}{\partial x}-\tau \frac{\partial}{\partial y}\right) .
$$

A map $f$ defined on a domain of $\mathbb{D}$ is said to be para-holomorphic if it satisfies $\frac{\partial f}{\partial \bar{z}}=0$. Observe that this is a hyperbolic equation, so a para-holomorphic map needs not to be analytic, and may be merely continuously differentiable.

On the Cartesian $n$-product $\mathbb{D}^{n}$ with para-complex coordinates $\left(z_{1}, \ldots, z_{n}\right)$, we define the canonical para-Kähler structure $\left(J_{*},\langle., .\rangle_{*}\right)$ on $\mathbb{D}^{n}$ by

$$
\begin{gathered}
J_{*}\left(z_{1}, \ldots, z_{n}\right):=\left(\tau z_{1}, \ldots, \tau z_{n}\right) \\
\langle., .\rangle_{*}:=\sum_{j=1}^{n} d z_{j} d \bar{z}_{j}=\sum_{j=1}^{n} d x_{j}^{2}-d y_{j}^{2} .
\end{gathered}
$$


We also introduce the "para-Hermitian" form:

$$
\langle\langle., .\rangle\rangle_{*}:=\sum_{j=1}^{n} d z_{j} \otimes d \bar{z}_{j}=\sum_{j=1}^{n} d x_{j}^{2}-d y_{j}^{2}-\tau \sum_{j=1}^{n} d x_{j} \wedge d y_{j} .
$$

In other words we recover $\langle., .\rangle_{*}$ by taking the real part of $\langle\langle., .\rangle\rangle_{*}$, while its imaginary part is minus the associated symplectic form $\omega:=\left\langle J_{*} ., .\right\rangle_{*}$.

\subsection{Pseudo- and para-Kähler manifolds}

Let $\mathcal{M}$ be a $2 n$-dimensional smooth manifold. An almost complex (resp. almost para-complex) structure $J$ is a $(1,1)$-tensor such that $J^{2}=-I d$ (resp. $\left.J^{2}=I d\right)$. In the almost para-complex case we furthermore require that the two eigen-distributions $\operatorname{Ker}(J \pm I d)$ have the same $\operatorname{rank}^{1}$. Since we shall always deal simultaneously with the two cases, we set $\epsilon$ equal to 1 in the complex case and -1 in the para-complex case, so that we have $J^{2}=-\epsilon I d$. Next we introduce the Nijenhuis tensor:

$$
N^{J}(X, Y):=[X, Y]-\epsilon([J X, J Y]-J[J X, Y]-J[X, J Y]) .
$$

The tensor $N^{J}$ vanishes if and only if $J$ is actually a complex (resp. para-complex structure), i.e. there exists an atlas on $\mathcal{M}$ whose transition maps are local bi-holomorphic (resp. bi-paraholomorphic) diffeomorphisms of $\mathbb{C}^{n}\left(\text { resp. } \mathbb{D}^{n}\right)^{2}$. A pseudo-Riemannian metric $g$ is said to be compatible with $J$ if

$$
g(J ., J .)=\epsilon g .
$$

A pair $(J, g)$, where $J$ is pseudo- or para-complex structure, and $g$ is a compatible pseudoRiemannian metric, is said to be a Kähler structure on $\mathcal{M}$ if the alternated 2-from $\omega:=\epsilon g(J .,$.$) is$ symplectic, i.e. $d \omega=0$. One may roughly summarize this by saying that pseudo- or para-Kähler geometry is the intersection of complex (or para-complex), pseudo-Riemannian and symplectic geometries.

\subsection{Volume minimization with respect to Hamiltonian variations}

Definition 1 Let $(\mathcal{M}, \omega)$ a 2n-dimensional symplectic manifold.

- A submanifold $\mathcal{L}$ of $\mathcal{M}$ is said to be Lagrangian if it has dimension $n$ and if the symplectic form $\omega$ vanishes on it.

- A vector field $X$ on $\mathcal{M}$ is said to be Hamiltonian if the 1 -form $X\lrcorner \omega$ is exact.

The relevance of Hamiltonian vector fields in the study of Lagrangian submanifolds is explained in the following proposition:

Proposition 1 Let $X$ be a Hamiltonian vector field on $\mathcal{M}$. Then the Lie derivative of $\omega$ along $X$ vanishes, and therefore if $\mathcal{L}$ is Lagrangian, so does $\exp _{t}(X)(\mathcal{L})$.

\footnotetext{
${ }^{1}$ In the almost complex case, the two eigen-distributions $\operatorname{Ker}(J \pm i I d)$ of $T \mathcal{M} \otimes \mathbb{C}$ have always the same rank.

${ }^{2}$ Observe that in the complex case, this is due to quite a hard result of Newlander and Nirenberg (see [NN]), while in the para-complex case it is a simple consequence of Frobenius theorem.
} 
Proposition $2(\mathcal{M}, J, g)$ be a pseudo- or para-Kähler manifold, $\mathcal{L}$ a non-degenerate Lagrangian submanifold and $X$ a compactly supported Hamiltonian vector field whose restriction to $\mathcal{L}$ is normal to $\mathcal{L}$. Then there exists $u \in C_{c}^{\infty}(\mathcal{L})$ such that $\left.X\right|_{\mathcal{L}}=J \nabla u$, where $\nabla$ denotes the gradient operator on $\mathcal{L}$ with respect to the induced metric.

We recall that the first variation formula states that

$$
\delta \mathcal{V}(\mathcal{L})(X):=\left.\frac{d}{d t} \mathcal{V}\left(\exp _{t}(X)(\mathcal{L})\right)\right|_{t=0}=-\int_{\mathcal{L}} g(n \vec{H}, X) d v
$$

(see the next section for the definition of the mean curvature $\vec{H}$ ).

Corollary 2 Let $(\mathcal{M}, J, g)$ be a pseudo- or para-Kähler manifold. Then a Lagrangian non-degenerate submanifold $\mathcal{L}$ is $H$-minimal, i.e. a critical point of the volume restricted to Hamiltonian variations, if and only if its mean curvature vector $\vec{H}$ satisfies the equation

$$
\operatorname{div}(n J \vec{H})=0,
$$

where div denotes the divergence operator on $\mathcal{L}$ with respect to the induced metric.

Definition 2 Let $(\mathcal{M}, J, g)$ be a pseudo- or para-Kähler manifold. A H-minimal Lagrangian submanifold $\mathcal{L}$ is said to be $\mathrm{H}$-stable if the quadratic form restricted to Hamiltonian variations $\delta^{2} \mathcal{V}(\mathcal{L})(X):=\left.\frac{d^{2}}{d t^{2}} \mathcal{V}\left(\exp _{t}(X)(\mathcal{L})\right)\right|_{t=0}$ is definite.

\subsection{The second fundamental form of a Lagrangian submanifold}

We recall briefly the definition of the second fundamental form of a submanifold $\mathcal{L}$ of $(\mathcal{M}, J, g)$ with non degenerate induced metric. At a point $x \in \mathcal{L} \subset \mathcal{M}$, we have the splitting $T_{x} \mathcal{M}=T_{x} \mathcal{L} \oplus N_{x} \mathcal{L}$ and for $X \in T_{x} \mathcal{M}$, we write $X=X^{\top}+X^{\perp}$ accordingly. We recall that the second fundamental form of $\mathcal{L}$ is defined by $h(X, Y)=\left(\nabla_{X} Y\right)^{\perp}$, where $\nabla$ denotes the Levi-Civita connection of $g$, and its shape operator by $A_{N} X=-\left(\nabla_{X} N\right)^{\top}$, where $\nabla^{\perp}$ is the connection of the normal bundle $N \mathcal{L}$. These two tensors carry actually the same information (the extrinsic geometry of $\mathcal{L}$ ) since they are related by the formula $g(h(X, Y), N)=g\left(A_{N} X, Y\right)$. The mean curvature $\vec{H}$ of $\mathcal{L}$ is the normal vector field obtained by tracing the second fundamental form $h$ with respect to the induced metric $g$.

If $\mathcal{L}$ is in addition Lagrangian, the formula $\omega=\epsilon g(J .,$.$) implies the J$ is an isometry (or antiisometry) of the tangent bundle $T \mathcal{L}$ of $\mathcal{L}$ onto its normal bundle $N \mathcal{L}$. This fact implies the following symmetry property:

Lemma 1 ([An2], [An4]) Let $\mathcal{L}$ be a non-degenerate, Lagrangian submanifold of a pseudo-Kähler or para-Kähler manifold $(\mathcal{M}, J, g)$. Then the map

$$
g(h(X, Y), J Z)=g\left(A_{J Z} X, Y\right)
$$

is tri-symmetric, i.e.

$$
g(h(X, Y), J Z)=g(h(Y, X), J Z)=g(h(X, Z), J Y) .
$$




\section{The second variation formula under Hamiltonian varia- tions (Proof of the Main Theorem)}

We start from the evolution equation satisfied by the volume density $d v$ associated to the induced metric

$$
\frac{d}{d t}(d v)=-g(n \vec{H}, X) d v
$$

which, in particular, implies the well-known first variation formula

$$
\frac{d}{d t} \mathcal{V}\left(\exp _{t}(X)(\mathcal{L})\right)=-\int_{\exp _{t}(X)(\mathcal{L})} g(n \vec{H}, X) d v
$$

Differentiating Equation 1 at $t=0$ yields

$$
\delta^{2} \mathcal{V}(\mathcal{L})(X)=-\left.\int_{\mathcal{L}} \frac{d}{d t}(g(n \vec{H}, X))\right|_{t=0} d v+\int_{\mathcal{L}} g(n \vec{H}, X)^{2} d v .
$$

Letting $\left(e_{1}, \ldots, e_{n}\right)$ be a frame which is orthonormal at $t=0$, we set $g_{i j}:=g\left(e_{i}, e_{j}\right)$ and $g^{i j}$ to be the coefficients of the inverse matrix of $\left[g_{i j}\right]_{1 \leq i, j \leq n}$. Using the Einstein sum convention, we have

$$
\begin{aligned}
\frac{d}{d t} g(n \vec{H}, X) & =\frac{d}{d t}\left(g^{i j} g\left(h\left(e_{i}, e_{j}\right), X\right)\right) \\
& =\frac{d g^{i j}}{d t} g\left(h\left(e_{i}, e_{j}\right), X\right)+g^{i j} g\left(\frac{d}{d t} h\left(e_{i}, e_{j}\right), X\right)+g^{i j} g\left(h\left(e_{i}, e_{j}\right), \frac{d X}{d t}\right) \\
& =\frac{d g^{i j}}{d t} g\left(h\left(e_{i}, e_{j}\right), X\right)+g^{i j} g\left(\frac{d}{d t} h\left(e_{i}, e_{j}\right), X\right)+g\left(n \vec{H}, \frac{d X}{d t}\right) .
\end{aligned}
$$

We deal with the first two terms of Equation 3 in the following Lemma:

Lemma 2 We have:

$\left.\frac{d g^{i j}}{d t}\right|_{t=0} g\left(h\left(e_{i}, e_{j}\right), X\right)+g^{i j} g\left(\left.\frac{d}{d t} h\left(e_{i}, e_{j}\right)\right|_{t=0}, X\right)=-g\left(\nabla^{\perp} X, \nabla^{\perp} X\right)-g\left(R^{\perp} X, X\right)+g\left(A_{X}, A_{X}\right)+\Delta f$,

where $f:=\frac{1}{2} g(X, X)$.

Proof. The proof is elementary and can be found in [An2] (Proof of Theorem 5, Chapter 1) ${ }^{3}$ or in $[\mathrm{Xi}])$.

The third term of Equation 3 does not vanish since $\mathcal{L}$ is not minimal, nor the vector field $\frac{d X}{d t}$ is Hamiltonian a priori:

Lemma 3 We have:

$$
\int_{\mathcal{L}} g\left(n \vec{H},\left.\frac{d X}{d t}\right|_{t=0}\right)=\epsilon \int_{\mathcal{L}} g(n \vec{H}, h(\nabla u, \nabla u))
$$

\footnotetext{
${ }^{3}$ The Reader is warned that although it is assumed in [An2] that $\vec{H}$ vanishes, this assumption is not used in the computation of the formula, which is therefore still valid here.
} 
Proof. Since the vector field $X(., t)$ is Hamiltonian $\forall t$, there exists $t_{0}>0$ and $\bar{u}(x, t) \in C_{c}^{\infty}(\mathcal{L} \times$ $\left.\left(-t_{0}, t_{0}\right)\right)$ such that $\bar{u}(., 0)=u$ and $X(., t)=J \nabla \bar{u}(., t)$. If follows that

$$
\begin{aligned}
\left.\frac{d X}{d t}\right|_{t=0} & =\left.\frac{d}{d t} g^{i j}\left(e_{i}(\bar{u}) J e_{j}\right)\right|_{t=0} \\
& =\left.\frac{d g^{i j}}{d t}\right|_{t=0} e_{i}(u) J e_{j}+\left.\epsilon_{i} \frac{d e_{i}(\bar{u})}{d t}\right|_{t=0} J e_{i}+\left.\epsilon_{i} e_{i}(u) \frac{d}{d t} J e_{i}\right|_{t=0} .
\end{aligned}
$$

Taking into account the fact that $\left.\frac{d g^{i j}}{d t}\right|_{t=0}=2 \epsilon_{i} \epsilon_{j} g\left(h\left(e_{i}, e_{j}\right), X\right)$ (cf Lemma 4, p. 24, [An2]) and the tri-symmetry of the curvature tensor $g(h(.,), J$.$) (Lemma 1)$, we first calculate

$$
\begin{aligned}
\left.\frac{d g^{i j}}{d t}\right|_{t=0} e_{i}(u) J e_{j} & =2 \epsilon_{i} \epsilon_{j} g\left(h\left(e_{i}, e_{j}\right), J \nabla u\right) e_{i}(u) J e_{j} \\
& =2 \epsilon_{i} \epsilon_{j} \epsilon_{k} g\left(h\left(e_{i}, e_{j}\right), J e_{k}\right) e_{k}(u) e_{i}(u) J e_{j} \\
& =2 \epsilon_{i} \epsilon_{j} \epsilon_{k} g\left(h\left(e_{i}, e_{k}\right), J e_{j}\right) e_{k}(u) e_{i}(u) J e_{j} \\
& =2 \epsilon_{j} g\left(h(\nabla u, \nabla u), J e_{j}\right) J e_{j} \\
& =2 \epsilon h(\nabla u, \nabla u) .
\end{aligned}
$$

On the other hand, using the fact that $\left[X, e_{i}\right]=0$ at $t=0$, we get

$$
\begin{aligned}
\left.\epsilon_{i} e_{i}(u) \frac{d}{d t} J e_{i}\right|_{t=0} & =\epsilon_{i} e_{i}(u) \nabla_{X} J e_{i} \\
& =\epsilon_{i} e_{i}(u) J \nabla_{e_{i}}^{\perp} X \\
& =-\epsilon_{i} e_{i}(u) J A_{X} e_{i} \\
& =-J A_{X} \nabla u,
\end{aligned}
$$

so that, using again Lemma 1:

$$
\begin{aligned}
g\left(n \vec{H},\left.\epsilon_{i} e_{i}(u) \frac{d}{d t} J e_{i}\right|_{t=0}\right) & =-g\left(n \vec{H}, J A_{J \nabla u} \nabla u\right) \\
& =g\left(J n \vec{H}, A_{J \nabla u} \nabla u\right) \\
& =g(h(J n \vec{H}, \nabla u), J \nabla u) \\
& =g(h(\nabla u, \nabla u), J(J n \vec{H})) \\
& =-\epsilon g(h(\nabla u, \nabla u), n \vec{H})) .
\end{aligned}
$$

We conclude, taking into account that the vector field $J \nabla\left(\left.\frac{\partial \bar{u}}{\partial t}\right|_{t=0}\right)$ is Hamiltonian,

$$
\begin{aligned}
\int_{\mathcal{L}} g\left(n \vec{H},\left.\frac{d X}{d t}\right|_{t=0}\right) & =\int_{\mathcal{L}} g\left(n \vec{H},\left.\frac{d g^{i j}}{d t}\right|_{t=0} e_{i}(u) J e_{j}+\left.\epsilon_{i} \frac{d e_{i}(\bar{u})}{d t}\right|_{t=0} J e_{i}+\left.\epsilon_{i} e_{i}(u) \frac{d}{d t} J e_{i}\right|_{t=0}\right) \\
& \left.=\int_{\mathcal{L}} 2 \epsilon g(n \vec{H}, h(\nabla u, \nabla u))+g\left(n \vec{H}, J \nabla\left(\left.\frac{\partial \bar{u}}{\partial t}\right|_{t=0}\right)\right)-\epsilon g(h(\nabla u, \nabla u), n \vec{H})\right) \\
& =\epsilon \int_{\mathcal{L}} g(n \vec{H}, h(\nabla u, \nabla u)),
\end{aligned}
$$

which is the claimed formula. 
Using Lemmas 2, 3 and Equation 3, the second variation formula (Equation 2) becomes

$$
\delta^{2} \mathcal{V}(\mathcal{L})=\int_{\mathcal{L}} g\left(\nabla^{\perp} X, \nabla^{\perp} X\right)+g\left(R^{\perp} X, X\right)-g\left(A_{X}, A_{X}\right)-\epsilon g(h(\nabla u, \nabla u), n \vec{H})+g(n \vec{H}, X)^{2} .
$$

The next Lemma relates two terms of the above expression to the Ricci tensors of $\mathcal{M}$ and $\mathcal{L}$ :

Lemma 4 Let $V$ be a tangent vector to $\mathcal{L}$, then the following formula holds:

$$
g\left(R^{\perp} J V, J V\right)-g\left(A_{J V}, A_{J V}\right)=\epsilon\left(-\operatorname{Ric}^{\mathcal{M}}(V, V)+\operatorname{Ric}^{\mathcal{L}}(V, V)-g(n \vec{H}, h(V, V))\right)
$$

where Ric ${ }^{\mathcal{M}}$ denotes the Ricci tensor of $(\mathcal{M}, g)$ and Ric ${ }^{\mathcal{L}}$ denotes the Ricci tensor of $\mathcal{L}$ endowed with the induced metric.

Proof: Observe first that

$$
\begin{aligned}
g\left(A_{J V}, A_{J V}\right) & =\sum_{i, j=1}^{n} \epsilon_{i} \epsilon_{j} g\left(A_{J V} e_{i}, e_{j}\right) \\
& =\sum_{i, j=1}^{n} \epsilon_{i} \epsilon_{j} g\left(h\left(e_{i}, e_{j}\right), J V\right) \\
& =\sum_{i, j=1}^{n} \epsilon_{i} \epsilon_{j} g\left(h\left(V, e_{i}\right), J e_{j}\right) \\
& =\epsilon \sum_{i, j=1}^{n} \epsilon_{i}\left(\epsilon \epsilon_{j}\right) g\left(h\left(V, e_{i}\right), J e_{j}\right) \\
& =\epsilon \sum_{i=1}^{n} g\left(h\left(V, e_{i}\right), h\left(V, e_{i}\right)\right) .
\end{aligned}
$$

We conclude, using Gauss equation for immersions and the fact that $\operatorname{Ric}^{\mathcal{M}}\left(J ., J_{.}\right)=\epsilon \operatorname{Ric}^{\mathcal{M}}(.,$.$) ,$

$$
\begin{aligned}
g\left(R^{\perp} J V, J V\right) & =\sum_{i=1}^{n} \epsilon_{i} g\left(R^{\mathcal{M}}\left(J V, e_{i}\right) J V, e_{i}\right) \\
& =\operatorname{Ric}^{M}(J V, J V)-\sum_{i=1}^{n} \epsilon \epsilon_{i} g\left(R^{\mathcal{M}}\left(J V, J e_{i}\right) J V, J e_{i}\right) \\
& =\epsilon \operatorname{Ric}^{\mathcal{M}}(V, V)-\sum_{i=1}^{n} \epsilon \epsilon_{i} g\left(R^{\mathcal{M}}\left(V, e_{i}\right) V, e_{i}\right) \\
& =\epsilon \operatorname{Ric}^{\mathcal{M}}(V, V)-\sum_{i=1}^{n} \epsilon \epsilon_{i}\left(g\left(R^{\mathcal{L}}\left(V, e_{i}\right) V, e_{i}\right)-g\left(h\left(e_{i}, e_{i}\right), h(V, V)\right)+g\left(h\left(V, e_{i}\right), h\left(V, e_{i}\right)\right)\right) \\
& =\epsilon \operatorname{Ric}^{\mathcal{M}}(V, V)-\epsilon \operatorname{Ric}^{\mathcal{L}}(V, V)-\epsilon g(n \vec{H}, h(V, V))+g\left(A_{J V}, A_{J V}\right) .
\end{aligned}
$$


We observe now that $g\left(\nabla^{\perp} J \nabla u, \nabla^{\perp} J \nabla u\right)=\epsilon g\left(\nabla^{2} u, \nabla^{2} u\right)$, where the Hessian operator $\nabla^{2} u$ is defined by $\nabla^{2} u(X, Y)=g\left(\nabla_{X} \nabla u, Y\right)=g\left(\nabla_{Y} \nabla u, X\right)$. Using Lemma 4, Equation 4, we get

$$
\delta^{2} \mathcal{V}(\mathcal{L})(J \nabla u)=\int_{\mathcal{L}} \epsilon\left(g\left(\nabla^{2} u, \nabla^{2} u\right)-R i c^{\mathcal{M}}(\nabla u, \nabla u)+R i c^{\mathcal{L}}(\nabla u, \nabla u)-2 g(n \vec{H}, h(\nabla u, \nabla u))\right)+g(n \vec{H}, J \nabla u)^{2} .
$$

The next step consists of extending a formula due to Reilly (see [Re]) to the pseudo-Riemannian setting:

Lemma 5 Let $(\mathcal{L}, g)$ be a pseudo-Riemannian manifold with Ricci tensor Ric ${ }^{\mathcal{L}}$ and $u$ a smooth, compactly supported function on $\mathcal{L}$. Then

$$
\int_{\mathcal{L}}(\Delta u)^{2}-g\left(\nabla^{2} u, \nabla^{2} u\right)=\int_{\mathcal{L}} \operatorname{Ric}^{\mathcal{L}}(\nabla u, \nabla u) .
$$

Proof. The proof is based on the generalization of Bochner's formula to the pseudo-Riemannian setting whose proof is postponed to the Appendix of this paper:

$$
\frac{1}{2} \Delta(g(\nabla u, \nabla u))=\operatorname{Ric}^{\mathcal{L}}(\nabla u, \nabla u)+g(\nabla u, \nabla(\Delta u))+g\left(\nabla^{2} u, \nabla^{2} u\right) .
$$

Integrating this equation over $\mathcal{L}$ yields

$$
\begin{aligned}
0=\int_{\mathcal{L}} \frac{1}{2} \Delta(g(\nabla u, \nabla u)) & =\int_{\mathcal{L}} \operatorname{Ric}^{\mathcal{L}}(\nabla u, \nabla u)+g(\nabla u, \nabla(\Delta u))+g\left(\nabla u^{2}, \nabla u^{2}\right) \\
& =\int_{\mathcal{L}} \operatorname{Ric}^{\mathcal{L}}(\nabla u, \nabla u)-(\Delta u)^{2}+g\left(\nabla u^{2}, \nabla u^{2}\right) .
\end{aligned}
$$

We are now in position to complete the proof of the Main theorem: applying Lemma 5 to Equation 5 gives

$$
\delta^{2} \mathcal{V}(\mathcal{L})(X)=\int_{\mathcal{L}} \epsilon\left((\Delta u)^{2}-R i c^{\mathcal{L}}(\nabla u, \nabla u)-2 g(n \vec{H}, h(\nabla u, \nabla u))\right)+g(n \vec{H}, J \nabla u)^{2},
$$

the required formula.

\section{Applications}

\subsection{Minimal Lagrangian submanifolds in para-Kähler-Einstein mani- folds (Proof of Theorem 1)}

In this section we assume that the Lagrangian submanifold $\mathcal{L}$ is not only $\mathrm{H}$-minimal but also minimal and that $(\mathcal{M}, g)$ is Einstein, i.e. there exists a real function $c$ such that $\operatorname{Ric}^{\mathcal{M}}=c g$. It is well known that $c$ must be constant and that the scalar curvature of $(\mathcal{M}, g)$ is $2 n c$. Then the second variation formula of the volume of $\mathcal{L}$ with respect Hamiltonian variations becomes:

$$
\begin{aligned}
\delta^{2} \mathcal{V}(\mathcal{L})(J \nabla u) & =\epsilon \int_{\mathcal{L}}(\Delta u)^{2}-\operatorname{Ric}^{\mathcal{M}}(\nabla u, \nabla u) \\
& =\epsilon \int_{\mathcal{L}}(\Delta u)^{2}-c g(\nabla u, \nabla u) \\
& =\epsilon \int_{\mathcal{L}}(\Delta u)^{2}+c u \Delta u
\end{aligned}
$$


Observe that this formula depends only on the induced metric on $\mathcal{L}$.

If we assume furthermore that $\mathcal{L}$ is compact and that its induced metric is definite, we may use the spectral properties of the Laplacian $-\Delta$ of a Riemannian metric: it is well known that $-\Delta$ is an elliptic operator and that the space $L^{2}(\mathcal{L})$ of square integrable real functions of $\mathcal{L}$ enjoys a Hilbertian basis $\left(\phi_{i}\right)_{i \in \mathbb{N}}$ satisfying $-\Delta \phi_{i}=\lambda_{i} \phi_{i}$, where $\left(\lambda_{i}\right)_{i \in \mathbb{N}}$ is an increasing sequence of strictly positive numbers tending to infinity. Hence, given $u=\sum_{i \in \mathbb{N}} a_{i} \phi_{i} \in C_{c}^{\infty}(\mathcal{L}) \subset L^{2}(\mathcal{L})$, we have $-\Delta u=\sum_{i \in \mathbb{N}} \lambda_{i} a_{i} \phi_{i}$. If follows that

$$
\begin{aligned}
\delta^{2} \mathcal{V}(\mathcal{L})(J \nabla u) & =\epsilon\left(\langle\Delta u, \Delta u\rangle_{L^{2}(\mathcal{L})}+c\langle u, \Delta u\rangle_{L^{2}(\mathcal{L})}\right) \\
& =\epsilon\left(\sum_{i \in \mathbb{N}} \lambda_{i}^{2} a_{i}^{2}-c \sum_{i \in \mathbb{N}} \lambda_{i} a_{i}^{2}\right) \\
& =\epsilon \sum_{i \in \mathbb{N}} a_{i}^{2} \lambda_{i}\left(\lambda_{i}-c\right) .
\end{aligned}
$$

Therefore, if $\lambda_{1} \geq c$, the second variation $\delta^{2} \mathcal{V}(\mathcal{L})(J \nabla u)$ has the sign of $\epsilon$ and $\mathcal{L}$ is H-stable.

Conversely, if $\lambda_{1}<c$, it easy to see that

$$
\epsilon \delta^{2} \mathcal{V}(\mathcal{L})\left(J \nabla \phi_{1}\right)=\lambda_{1}\left(\lambda_{1}-c\right)<0,
$$

while, since $\lim _{i \rightarrow \infty} \lambda_{i}=\infty$, there exists $i_{0}$ such that

$$
\epsilon \delta^{2} \mathcal{V}(\mathcal{L})\left(J \nabla \phi_{i_{0}}\right)=\lambda_{i_{0}}\left(\lambda_{i_{0}}-c\right)>0 .
$$

Hence $\mathcal{L}$ is $\mathrm{H}$-unstable.

In the para-Kähler case, we get Theorem 1 . On the other hand, if $(\mathcal{M}, J, g)$ is pseudo-Kähler with signature $(2 n, 2(n-p))$, the induced metric on $\mathcal{L}$ has signature $(n, n-p)$. Hence the discussion above applies only in the Kähler case. We therefore recover exactly Oh's stability criterion:

Stability Criterion $([\mathrm{Oh} 1])$ : Let $(\mathcal{M}, J, g)$ be an Einstein-Kähler manifold with with scalar curvature 2 nc and $\mathcal{L}$ is a compact, minimal, Lagrangian submanifold of $\mathcal{M}$. Let $\lambda_{1}$ be the first eigenvalue of the Laplacian. Then $\mathcal{L}$ is $H$-stable if and only if $\lambda_{1}>c$.

\subsection{Tori $\mathbb{T}_{r_{1}, \ldots, r_{n}}^{n}$ are H-unstable (proof of Theorem 2)}

We endow the complex vector space $\mathbb{C}^{n}$ with the Hermitian form

$$
\langle\langle., .\rangle\rangle_{p}:=\sum_{j=1}^{n} \epsilon_{j} d z_{j} \otimes d \bar{z}_{j}=\langle., .\rangle_{p}-i \omega_{p}
$$

where $\epsilon_{j}= \pm 1$. A parametrization of the torus $\mathbb{T}_{r_{1}, \ldots, r_{n}}^{n}:=\mathbb{S}^{1}\left(r_{1}\right) \times \ldots \times \mathbb{S}^{1}\left(r_{n}\right) \subset \mathbb{C}^{n}$ is

$$
\begin{array}{ccc}
f: \mathbb{R} / 2 \pi r_{1} \mathbb{Z} \times \ldots \times \mathbb{R} / 2 \pi r_{n} \mathbb{Z} & \rightarrow & \mathbb{C}^{n} \\
\left(s_{1}, \ldots, s_{n}\right) & \mapsto & \left(r_{1} \exp \left(i s_{1} / r_{1}\right), \ldots, r_{n} \exp \left(i s_{n} / r_{n}\right)\right) .
\end{array}
$$

The first derivatives of $f$ are

$$
f_{s_{j}}=i \exp \left(i s_{j} / r_{j}\right) e_{j}
$$


where $\left(e_{1}, \ldots, e_{n}\right)$ is the canonical Hermitian basis of $\mathbb{C}^{n}$. It is straightforward to check that $f$ is Lagrangian with respect to $\omega_{p}$ and that the induced metric $f^{*}\langle., .\rangle_{2 p}$ takes the form $\sum_{j=1}^{n} \epsilon_{j} d s_{j}^{2}$. In particular it is non-degenerate and flat.

Moreover, setting

$$
N_{j}:=J f_{s_{j}}=-\exp \left(i s_{j} / r_{j}\right) e_{j}
$$

we have

$$
h_{j k l}:=\left\langle f_{s_{j} s_{k}}, N_{l}\right\rangle_{2 p}=\frac{\epsilon_{j} \delta_{j k} \delta_{k l}}{r_{j}} .
$$

Therefore

$$
\left\langle n \vec{H}, N_{j}\right\rangle_{2 p}=\sum_{k=1}^{n} \epsilon_{k} h_{j k k}=\frac{1}{r_{j}}
$$

and

$$
n \vec{H}=\sum_{j=1}^{n} \frac{\epsilon_{j}}{r_{j}} N_{j}
$$

In particular, $\operatorname{div}(n J \vec{H})$ vanishes, i.e. $f$ is H-minimal.

On the other hand, letting $u$ be a smooth map on $\mathbb{T}_{r_{1}, \ldots, r_{n}}^{n}$, we have $\nabla u=\sum_{j=1}^{n} \epsilon_{j} u_{s_{j}} \partial_{s_{j}}$, so $J \nabla u=-\sum_{j=1}^{n} \epsilon_{j} u_{s_{j}} N_{j}$. It follows that

$$
\langle n \vec{H}, J \nabla u\rangle_{2 p}=-\sum_{j=1}^{n} \frac{u_{s_{j}}}{r_{j}}
$$

and

$$
\begin{aligned}
\langle h(\nabla u, \nabla u), n \vec{H}\rangle_{2 p} & =\sum_{j=1}^{n} u_{s_{j}}^{2}\left\langle h\left(\partial_{s_{j}}, \partial_{s_{j}}\right), 2 \vec{H}\right\rangle_{2 p} \\
& =\sum_{j=1}^{n} u_{s_{j}}^{2} \frac{h_{j j j}}{r_{j}} \\
& =\sum_{j=1}^{n} u_{s_{j}}^{2} \frac{u_{s_{j}}^{2}}{r_{j}^{2}} .
\end{aligned}
$$

Finally, applying the Main Theorem,

$$
\begin{aligned}
\delta^{2} \mathcal{V}\left(\mathbb{T}_{r_{1}, \ldots, r_{n}}^{n}\right)(J \nabla u) & =\int_{\mathbb{R} / 2 \pi r_{1} \mathbb{Z} \times \ldots \times \mathbb{R} / 2 \pi r_{n} \mathbb{Z}}\left(\left(\sum_{j=1}^{n} \epsilon_{j} u_{s_{j} s_{j}}\right)^{2}-2 \sum_{j=1}^{n} \frac{u_{s_{j}}^{2}}{r_{j}^{2}}+\left(\sum_{j=1}^{n} \epsilon_{j} \frac{u_{s_{j}}}{r_{j}}\right)^{2}\right) d s_{1} \ldots d s_{n} \\
& =\int_{\mathbb{R} / 2 \pi r_{1} \mathbb{Z} \times \ldots \times \mathbb{R} / 2 \pi r_{n} \mathbb{Z}}\left(\left(\sum_{j=1}^{n} \epsilon_{j} u_{s_{j} s_{j}}\right)^{2}-\sum_{j=1}^{n} \frac{u_{s_{j}}^{2}}{r_{j}^{2}}+2 \sum_{j<k} \epsilon_{j} \epsilon_{k} \frac{u_{s_{j}} u_{s_{k}}}{r_{j} r_{k}}\right) d s_{1} \ldots d s_{n} .
\end{aligned}
$$

From this expression, it is easy to check that the second variation is indefinite, exhibiting both positive and negative directions: 
- On the one hand, taking $u\left(s_{1}, \ldots, s_{n}\right)=\cos \left(k s_{1} / r_{1}\right)$, where $k$ is an arbitrary integer, we get

$$
\begin{aligned}
\delta \mathcal{V}^{2}\left(\mathbb{T}_{r_{1}, \ldots, r_{2}}^{n}\right)(J \nabla u) & =\left(\prod_{j=2}^{n} 2 \pi r_{j}\right)\left(\frac{k^{4}}{r_{1}^{4}} \int_{0}^{2 \pi r_{1}}\left(\cos \left(k s_{1} / r_{1}\right)\right)^{2} d s_{1}-\frac{k^{2}}{r_{1}^{4}} \int_{0}^{2 \pi r_{1}}\left(\sin \left(k s_{1} / r_{1}\right)\right)^{2} d s_{1}\right) \\
& =\left(\prod_{j=2}^{n} 2 \pi r_{j}\right) \frac{\pi\left(k^{4}-k^{2}\right)}{r_{1}^{3}}
\end{aligned}
$$

which is strictly positive if $k>1$.

- On the other hand, since $p \neq 0, n$, there exists both positive and negative vectors in the canonical basis $\left(e_{1}, \ldots, e_{n}\right)$. Assume, without loss of generality, that $\epsilon_{1}=1$ and $\epsilon_{2}=-1$. Consider first the case $\frac{r_{1}}{r_{2}} \in \mathbb{Q}$; we shall use the fact that the wave equation $u_{s_{1} s_{1}}-u_{s_{2} s_{2}}=0$ admits globally defined solutions: given a $2 \pi$-periodic, non constant map $F$, and two integers $a$ and $b$ satisfying $\frac{a}{b}=\frac{r_{1}}{r_{2}}$, we set $u\left(s_{1}, \ldots, s_{n}\right):=F\left(\frac{a s_{1}}{r_{1}}-\frac{b s_{2}}{r_{2}}\right)$. We then get

$$
\sum_{j=1}^{n} \epsilon_{j} u_{s_{j} s_{j}}=u_{s_{1} s_{1}}-u_{s_{2} s_{2}}=\left(\frac{a^{2}}{r_{1}^{2}}-\frac{b^{2}}{r_{2}^{2}}\right) F^{\prime \prime}=0
$$

and

$$
-\sum_{j=1}^{n} \frac{u_{s_{j}}^{2}}{r_{j}^{2}}+2 \sum_{j<k} \epsilon_{j} \epsilon_{k} \frac{u_{s_{j}} u_{s_{k}}}{r_{j} r_{k}}=-\left(\frac{u_{s_{1}}}{r_{1}}+\frac{u_{s_{2}}}{r_{2}}\right)^{2}=-\left(\frac{a}{r_{1}^{2}}+\frac{b}{r_{2}^{2}}\right)^{2}\left(F^{\prime}\right)^{2} .
$$

Hence

$$
\delta^{2} \mathcal{V}\left(\mathbb{T}_{r_{1}, \ldots, r_{2}}^{n}\right)(J \nabla u)=-\prod_{j=3}^{n}\left(2 \pi r_{j}\right)\left(\frac{a}{r_{1}^{2}}+\frac{b}{r_{2}^{2}}\right)^{2} \int_{\mathbb{R} / 2 \pi r_{1} \mathbb{Z} \times \mathbb{R} / 2 \pi r_{2}}\left(F^{\prime}\right)^{2} d s_{1} d s_{2}<0 .
$$

To conclude, observe we have proved H-instability of $\mathbb{T}_{r_{1}, \ldots, r_{n}}^{n}$ for a dense set of values of $\frac{r_{1}}{r_{2}}$. The H-instability being an open condition, it follows that all tori $\mathbb{T}_{r_{1}, \ldots, r_{n}}^{n}$ are H-unstable.

\subsection{Stability and instability of the products $\mathbb{H}_{r_{1}, \ldots, r_{n}}^{n}$ (proof of Theorem 3)}

We refer to Section 1.1 for notation and introduce furthermore:

$$
\exp \epsilon(\tau t):= \begin{cases}\cosh (t)+\tau \sinh (t) & \text { if } \quad \epsilon=1 \\ \sinh (t)+\tau \cosh (t) & \text { if } \quad \epsilon=-1\end{cases}
$$

Observe that $\exp \epsilon(\tau t)^{\prime}=\exp (-\epsilon)(\tau t)=\tau \exp \epsilon(\tau t)$ and that $|\exp \epsilon(\tau t)|^{2}=\epsilon$. A parametrization of $\mathbb{H}_{r_{1}, \ldots, r_{n}}^{n}$ is:

$$
\begin{array}{rlrl}
f: & \mathbb{R}^{n} & \rightarrow & \mathbb{D}^{n} \\
\left(s_{1}, \ldots, s_{n}\right) & \mapsto & \left(r_{1} \exp \epsilon_{1}\left(\tau s_{1} / r_{1}\right), \ldots, r_{n} \exp \epsilon_{n}\left(\tau s_{n} / r_{n}\right)\right) .
\end{array}
$$

The first derivatives of $f$ are

$$
f_{s_{j}}=\tau \exp \epsilon_{j}\left(\tau s_{j} / r_{j}\right) e_{j}
$$

where $\left(e_{1}, \ldots, e_{n}\right)$ is the canonical para-Hermitian basis of $\mathbb{D}^{n}$. It is straightforward to check that $f$ is Lagrangian with respect to $\omega_{*}$ and that the induced metric $f^{*}\langle., .\rangle_{*}$ takes the form $-\sum_{j=1}^{n} \epsilon_{j} d s_{j}^{2}$. In particular it is non-degenerate and flat. 
Next, setting $N_{j}:=J_{*} f_{s_{j}}=\exp \epsilon_{j}\left(\tau s_{j} / r_{j}\right) e_{j}$, we easily calculate the mean curvature vector of $f$ :

$$
n \vec{H}=-\sum_{j=1}^{n} \frac{\epsilon_{j}}{r_{j}} N_{j}
$$

In particular, $\operatorname{div}\left(n J_{*} \vec{H}\right)$ vanishes, i.e. $f$ is H-minimal.

On the other hand, letting $u$ be a compactly supported smooth map on $\mathbb{H}_{r_{1}, \ldots, r_{n}}$, we have $\nabla u=$ $-\sum_{j=1}^{n} \epsilon_{j} u_{s_{j}} \partial_{s_{j}}$, so $J_{*} \nabla u=-\sum_{j=1}^{n} \epsilon_{j} u_{s_{j}} N_{j}$. It follows that

$$
\left\langle n \vec{H}, J_{*} \nabla u\right\rangle_{*}=\sum_{j=1}^{n} \frac{\epsilon_{j} u_{s_{j}}}{r_{j}}
$$

and

$$
\begin{aligned}
\langle h(\nabla u, \nabla u), n \vec{H}\rangle_{*} & =\sum_{j=1}^{n} u_{s_{j}}^{2}\left\langle h\left(\partial_{s_{j}}, \partial_{s_{j}}\right), n \vec{H}\right\rangle_{*} \\
& =-\sum_{j=1}^{n} u_{s_{j}}^{2} \frac{\epsilon_{j} h_{j j j}}{r_{j}} \\
& =-\sum_{j=1}^{n} \frac{u_{s_{j}}^{2}}{r_{j}^{2}} .
\end{aligned}
$$

Hence, by the Main Theorem,

$$
\begin{aligned}
\delta^{2} \mathcal{V}\left(\mathbb{H}_{r_{1}, \ldots, r_{n}}^{n}\right)\left(J_{*} \nabla u\right) & =\int_{\mathbb{R}^{n}}\left(-\left(\sum_{j=1}^{n} \epsilon_{j} u_{s_{j} s_{j}}\right)^{2}-2 \sum_{j=1}^{n} \frac{u_{s_{j}}^{2}}{r_{j}^{2}}+\left(\sum_{j=1}^{n} \epsilon_{j} \frac{u_{s_{j}}}{r_{j}}\right)^{2}\right) d s_{1} \ldots d s_{n} \\
& =\int_{\mathbb{R}^{n}}\left(-\left(\sum_{j=1}^{n} \epsilon_{j} u_{s_{j} s_{j}}\right)^{2}-\sum_{j=1}^{n} \frac{u_{s_{j}}^{2}}{r_{j}^{2}}+2 \sum_{j<k} \epsilon_{j} \epsilon_{k} \frac{u_{s_{j}} u_{s_{k}}}{r_{j} r_{k}}\right) d s_{1} \ldots d s_{n} .
\end{aligned}
$$

In the one-dimensional case, this expressions becomes

$$
\delta^{2} \mathcal{L}\left(\mathbb{H}_{ \pm}^{1}\left(r_{1}\right)\right)\left(J_{*} \nabla u\right)=\int_{\mathbb{R}}\left(-\left(u^{\prime \prime}\right)^{2}-\left(\frac{u^{\prime}}{r_{1}}\right)^{2}\right) d s_{1} \leq 0,
$$

while in the two-dimensional case, we have

$$
\delta^{2} \mathcal{A}\left(\mathbb{H}_{r_{1}, r_{2}}^{2}\right)\left(J_{*} \nabla u\right)=\int_{\mathbb{R}^{2}}\left(-\left(\epsilon_{1} u_{s_{1} s_{1}}+\epsilon_{2} u_{s_{2} s_{2}}\right)^{2}-\left(\epsilon_{1} \frac{u_{s_{1}}}{r_{1}}-\epsilon_{2} \frac{u_{s_{2}}}{r_{2}}\right)^{2}\right) d s_{1} d s_{2} \leq 0 .
$$

Hence in dimension 1 and 2, the products $\mathbb{H}_{r_{1}, \ldots, r_{n}}^{n}$ are H-stable. We now prove that it is not anymore the case if $n \geq 3$. This follows from three elementary lemmas. For sake of brevity we set $s=\left(s_{1}, \ldots, s_{n}\right)$ and $d s=d s_{1} \ldots d s_{n}$. 


\section{Lemma 6 Set}

$$
Q(\nabla u, \nabla u):=\sum_{j=1}^{n} \frac{u_{s_{j}}^{2}}{r_{j}^{2}}-2 \sum_{j<k} \epsilon_{j} \epsilon_{k} \frac{u_{s_{j}} u_{s_{k}}}{r_{j} r_{k}} .
$$

If the quadratic functional

$$
\boldsymbol{Q}(u):=\int_{\mathbb{R}^{n}} Q(\nabla u, \nabla u) d s
$$

is indefinite, then the second variation

$$
\delta^{2} \mathcal{V}\left(\mathbb{H}_{r_{1}, \ldots, r_{n}}^{n}\right)\left(J_{*} \nabla u\right)=-\int_{\mathbb{R}^{n}}(\Delta u)^{2} d s-\int_{\mathbb{R}^{n}} Q(\nabla u, \nabla u) d s
$$

is indefinite as well.

Proof. Given $u \in C_{c}^{\infty}\left(\mathbb{R}^{n}\right)$, set $u^{t}(s):=t^{n / 2-1} u(t s)$. A quick calculation, using that $u_{s_{i}}^{t}=t^{n / 2} u_{s_{i}}$ and $u_{s_{i} s_{i}}^{t}=t^{n / 2+1} u_{s_{i} s_{i}}$, shows that

$$
\delta^{2} \mathcal{V}\left(\mathbb{H}_{r_{1}, \ldots, r_{n}}^{n}\right)\left(J_{*} \nabla u^{t}\right)=-t^{2} \int_{\mathbb{R}^{n}}(\Delta u)^{2} d s+\int_{\mathbb{R}^{n}} Q(\nabla u, \nabla u) d s
$$

Letting $t$ tends to 0 makes this expression to have the same sign than $\mathbf{Q}(u)$, so the indefiniteness of $\mathbf{Q}$ implies that of $\delta^{2} \mathcal{V}\left(\mathbb{H}_{r_{1}, \ldots, r_{n}}^{n}\right)$.

Lemma 7 There exists a linear change of variable $\sigma(s)$ and an integer $k, 1 \leq k \leq n-1$, such that

$$
\boldsymbol{Q}(u):=c \int_{\mathbb{R}^{n}}\left(\sum_{i=1}^{k} u_{\sigma_{i}}^{2}-\sum_{i=k+1}^{n} u_{\sigma_{i}}^{2}\right) d \sigma
$$

where $c$ is a non vanishing constant.

Proof. Observe that

$$
Q(\nabla u, \nabla u)=[\nabla u] \cdot M \cdot[\nabla u]^{\mathrm{T}},
$$

where the matrix $M$ is defined by

$$
M:=\operatorname{diag}\left(1 / r_{1}^{2}, \ldots, 1 / r_{n}^{2}\right)-\left[\frac{\epsilon_{i} \epsilon_{j}}{r_{i} r_{j}}\right]_{1 \leq i, j \leq n} .
$$

On the one hand, we have

$$
(1,0, \ldots, 0) \cdot M \cdot(1,0, \ldots, 0)^{\mathrm{T}}=\frac{1}{r_{i}^{2}}>0
$$

and on the other hand, $-(n-2)$ is an eigenvalue of $M$, with eigenvector $V=\left(\frac{\epsilon_{1}}{r_{1}}, \ldots, \frac{\epsilon_{n}}{r_{n}}\right)$, so

$$
V \cdot M \cdot V^{\mathrm{T}}=-(n-2)|V|_{0}^{2}<0 .
$$

Therefore the matrix $M$ is indefinite. The conclusion follows from Sylvester's law of inertia. 
Lemma 8 The quadratic functional

$$
\boldsymbol{Q}(u):=\int_{\mathbb{R}^{n}}\left(\sum_{i=1}^{k} u_{\sigma_{i}}^{2}-\sum_{i=k+1}^{n} u_{\sigma_{i}}^{2}\right) d \sigma
$$

is indefinite on the space of compactly supported, smooth functions $C_{c}^{\infty}\left(\mathbb{R}^{n}\right)$.

Proof. Given a non vanishing $u \in C_{c}^{\infty}\left(\mathbb{R}^{n}\right)$, set $u^{t}\left(\sigma_{1}, \ldots, \sigma_{n}\right):=u\left(t \sigma_{1}, \ldots, t \sigma_{k}, \sigma_{k+1}, \ldots, \sigma_{n}\right)$. A quick calculation shows that

$$
\mathbf{Q}\left(u^{t}\right):=t^{-k}\left(t^{2} \int_{\mathbb{R}^{n}}\left(\sum_{i=1}^{k} u_{\sigma_{i}}^{2}\right) d \sigma-\int_{\mathbb{R}^{n}}\left(\sum_{i=k+1}^{n} u_{\sigma_{i}}^{2}\right) d \sigma\right) .
$$

Since both integrals are non zero, letting $t$ tend to $\infty$ and 0 give respectively a positive and negative value to $\mathbf{Q}$.

\subsection{Minimal Lagrangian surfaces in the space of oriented geodesics of 3-dimensional space forms}

We briefly recall the construction of the canonical pseudo- or para-Kähler structures of the space of geodesics of the 3-dimensional space forms. For further detail, see [An4]. Consider first the flat pseudo-Riemannian metric $\langle\cdot, \cdot\rangle_{p}$ of $\mathbb{R}^{4}$ of signature $(p, 4-p)$ :

$$
\langle\cdot, \cdot\rangle_{p}:=-\sum_{i=1}^{p} d x_{i}^{2}+\sum_{i=p+1}^{4} d x_{i}^{2},
$$

and the 3-dimensional quadric

$$
\mathbb{S}_{p}^{3}=\left\{x \in \mathbb{R}^{4} \mid\langle x, x\rangle_{p}=1\right\} .
$$

The space $L^{+}\left(\mathbb{S}_{p}^{3}\right)$ (resp. $\left.L^{-}\left(\mathbb{S}_{p}^{3}\right)\right)$ of positive (resp. negative) oriented geodesics of the space form $\mathbb{S}_{p}^{3}$ can be identified with the Grassmannian $G r^{+}(4,2)$ (resp. $\left.G r^{+}(4,2)\right)$ of oriented two-planes of $\mathbb{R}^{4}$ with positive induced metric (resp. indefinite induced metric). Such Grassmannians, which are 4-dimensional, may be naturally embedded in the space of bi-vectors, a 6 -dimensional real linear space:

$$
\iota: L^{ \pm}\left(\mathbb{S}_{p}^{3}\right) \rightarrow \Lambda^{2}\left(\mathbb{R}^{4}\right)=\operatorname{Span}\left\{e_{i} \wedge e_{j}: 1 \leq i<j \leq 4\right\},
$$

where $\left(e_{1}, e_{2}, e_{3}, e_{4}\right)$ denotes the canonical basis of $\mathbb{R}^{4}$. We endow $\Lambda^{2}\left(\mathbb{R}^{4}\right)$ with the flat pseudoRiemannian metric

$$
\left\langle\left\langle x \wedge y, x^{\prime} \wedge y^{\prime}\right\rangle\right\rangle=\left\langle x, x^{\prime}\right\rangle_{p}\left\langle y, y^{\prime}\right\rangle_{p}-\left\langle x, y^{\prime}\right\rangle_{p}\left\langle y, x^{\prime}\right\rangle_{p}
$$

A tangent vector to $\iota\left(L^{ \pm}\left(\mathbb{S}_{p}^{3}\right)\right)$ at $x \wedge y$ takes the form $x \wedge X+y \wedge Y$, where $X, Y$ belong to the orthogonal $(x \wedge y)^{\perp}$ of $x \wedge y$. Any of the two 2-dimensional, oriented planes $x \wedge y$ and $(x \wedge y)^{\perp}$ is endowed with a natural complex or para-complex structure that we shall denote by $\mathrm{J}$ and $\mathrm{J}^{\prime}$ respectively. For example $\mathrm{J}$ is defined by $\mathrm{J} x:=y$ and $\mathrm{J} y:=-\epsilon x$, where $\epsilon:=\langle y, y\rangle_{p}$. 
We are now in position to define the two structures $(\mathbb{J}, \mathbb{G})$ and $\left(\mathbb{J}^{\prime}, \mathbb{G}^{\prime}\right)$. We first set $\mathbb{G}$ to be the induced metric on $L^{ \pm}\left(\mathbb{S}_{p}^{3}\right)$, i.e., $\mathbb{G}:=\iota^{*}\langle\langle.,\rangle$.$\rangle . Next we set \mathbb{J}$ and $\mathbb{J}^{\prime}$ to be respectively:

$$
\mathbb{J}(x \wedge X+y \wedge Y):=\mathrm{J} x \wedge X+\mathrm{J} y \wedge Y .
$$

and

$$
\mathbb{J}^{\prime}(x \wedge X+y \wedge Y):=x \wedge \mathrm{J}^{\prime} X+y \wedge \mathrm{J}^{\prime} Y .
$$

Finally, the neutral metric $\mathbb{G}^{\prime}$ is defined by

$$
\mathbb{G}^{\prime}:=\epsilon \mathbb{G}\left(\mathbb{J} ., \mathbb{J}^{\prime} .\right):=-\epsilon \mathbb{G}\left(., \mathbb{J} \circ \mathbb{J}^{\prime} .\right) .
$$

This implies that the two pseudo- or para-Kähler structures $(\mathbb{J}, \mathbb{G})$ and $\left(\mathbb{J}^{\prime}, \mathbb{G}^{\prime}\right)$ share the same symplectic form $\omega=\epsilon \mathbb{G}(\mathbb{J} .,)=.\epsilon^{\prime} \mathbb{G}^{\prime}\left(\mathbb{J}^{\prime} .\right.$, . .). Moreover the normal congruence of an oriented surface $\mathcal{S}$ of $\mathbb{S}_{p}^{3}$, i.e. the set of geodesics normal to $\mathcal{S}$, is a Lagrangian surface, and if $\mathcal{S}$ is an equidistant tube over a geodesic, its normal congruence is minimal with respect to both the metric $\mathbb{G}$ and $\mathbb{G}^{\prime}$ (see $[$ An4]). In the remainder of the section we shall discuss the H-stability of such surfaces with respect to $\mathbb{G}$ and $\mathbb{G}^{\prime}$.

Let $\gamma$ be a non-null geodesic of $\mathbb{S}_{p}^{3}$, with arclength parameter $s$ and $\left(n_{1}, n_{2}\right)$ a local, orthonormal frame of its normal bundle (hence $\left(\gamma, \gamma^{\prime}, n_{1}, n_{2}\right) \in S O(p, n-p)$ ) such that $D_{\gamma^{\prime}} n_{i}=n_{i}(s)^{\prime}=0$. The topological type of the tube over $\gamma$ depends on the type of the geodesic $\gamma$ and on the causal character of its normal space. In order to simplify the exposition, we set $\epsilon_{1}:=\left\langle\gamma^{\prime}, \gamma^{\prime}\right\rangle_{p}$ and $\epsilon_{4}:=\left\langle n_{1}, n_{1}\right\rangle_{p}\left\langle n_{2}, n_{2}\right\rangle_{p}$ (it will be clear in a moment that this apparently unnatural notation is chosen to match that of $[\mathrm{An} 4])$. Then the tube over $\gamma$ is topologically $\mathbb{S}_{\epsilon_{1}}^{1} \times \mathbb{S}_{\epsilon_{4}}^{1}$, where

$$
\mathbb{S}_{\epsilon}^{1}:=\{(\cos \epsilon(\sigma), \sin \epsilon(\sigma)) \mid \sigma \in \mathbb{R}\},
$$

i.e. a circle if $\epsilon=1$ and a hyperbola if $\epsilon=-1$. Hence a local parametrization of the tube over $\gamma$ of radius $\theta$ is is

$$
\begin{aligned}
\phi: \mathbb{S}_{\epsilon_{1}}^{1} \times \mathbb{S}_{\epsilon_{4}}^{1} & \rightarrow \\
(s, t) & \mapsto \cos \epsilon(\theta) \gamma+\sin \epsilon(\theta)\left(\cos \epsilon_{4}(t) n_{1}+\sin \epsilon_{4}(t) n_{2}\right) .
\end{aligned}
$$

Introducing

$$
e_{1}:=\gamma^{\prime} \quad e_{2}:=-\epsilon_{4} \sin \epsilon_{4}(t) n_{1}+\cos \epsilon_{4}(t) n_{2}
$$

we have

$$
\begin{aligned}
& \phi_{s}=\cos \epsilon(\theta) \gamma^{\prime}=\cos \epsilon(\theta) e_{1} \\
& \phi_{t}=\sin \epsilon(\theta)\left(-\epsilon_{4} \sin \epsilon_{4}(t) n_{1}+\cos \epsilon_{4}(t) n_{2}\right)=\sin \epsilon(\theta) e_{2} .
\end{aligned}
$$

On the other hand, a unit normal vector is given by

$$
N=-\epsilon \sin \epsilon(\theta) \gamma+\cos \epsilon(\theta)\left(\cos \epsilon_{4}(t) n_{1}+\sin \epsilon_{4}(t) n_{2}\right) .
$$

Observe that $\langle N, N\rangle_{p}=\left\langle n_{1}, n_{1}\right\rangle_{p}=\epsilon$. Next we have

$$
\begin{aligned}
& N_{s}=-\epsilon \sin \epsilon(\theta) \gamma^{\prime}=-\epsilon \sin \epsilon(\theta) e_{1} \\
& N_{t}=\cos \epsilon(\theta)\left(-\epsilon_{4} \sin \epsilon_{4}(t) n_{1}+\cos \epsilon_{4}(t) n_{2}\right)=\cos \epsilon(\theta) e_{2}
\end{aligned}
$$


The normal congruence of $\phi$ is parametrized by $\bar{\phi}:=\phi \wedge N$ (see [An4]). Setting

$$
\begin{aligned}
E_{1}:=\phi \wedge e_{1} & E_{2}:=\phi \wedge e_{2} \\
E_{3}:=N \wedge e_{1} & E_{4}:=N \wedge e_{2},
\end{aligned}
$$

we have

$$
\begin{gathered}
\bar{\phi}_{s}=\phi_{s} \wedge N+\phi \wedge N_{s}=-\cos \epsilon(\theta) E_{3}-\epsilon \sin \epsilon(\theta) E_{1} \\
\bar{\phi}_{t}=\phi_{t} \wedge N+\phi \wedge N_{t}=-\sin \epsilon(\theta) E_{4}+\cos \epsilon(\theta) E_{2} .
\end{gathered}
$$

Since the coefficients of the metrics $\mathbb{G}$ and $\mathbb{G}^{\prime}$ in the basis $\left(E_{1}, E_{2}, E_{3}, E_{4}\right)$ are

$$
\mathbb{G}=\operatorname{diag}\left(\epsilon_{1}, \epsilon^{\prime} \epsilon_{1}, \epsilon \epsilon_{1}, \epsilon \epsilon^{\prime} \epsilon_{1}\right):=\left(\epsilon_{1}, \epsilon_{2}, \epsilon_{3}, \epsilon_{4}\right)
$$

and

$$
\mathbb{G}^{\prime}=\left(\begin{array}{cccc}
0 & 0 & 0 & \epsilon_{2} \\
0 & 0 & -\epsilon_{2} & 0 \\
0 & -\epsilon_{2} & 0 & 0 \\
\epsilon_{2} & 0 & 0 & 0
\end{array}\right),
$$

the coefficient of induced metrics $\bar{\phi}^{*}(\mathbb{G})$ and $\bar{\phi}^{*}\left(\mathbb{G}^{\prime}\right)$ in the coordinates $(s, t)$ are

$$
\left(\begin{array}{cc}
\epsilon_{3} & 0 \\
0 & \epsilon_{2}
\end{array}\right) \text { and } \quad\left(\begin{array}{cc}
0 & \epsilon_{2} \\
\epsilon_{2} & 0
\end{array}\right) \text {. }
$$

Moreover, the coefficients of the restriction of the Ricci tensor $\bar{\phi}^{*}\left(\operatorname{Ric}^{\mathbb{G}}\right)$ and $\bar{\phi}^{*}\left(\operatorname{Ric}^{\mathbb{G}^{\prime}}\right)$ in the coordinates $(s, t)$ are

$$
2\left(\begin{array}{cc}
\epsilon_{1} & 0 \\
0 & \epsilon_{4}
\end{array}\right) \quad \text { and } \quad-2\left(\begin{array}{cc}
\epsilon_{1} & 0 \\
0 & \epsilon_{4}
\end{array}\right)
$$

We deduce that the Hamiltonian second variation of the area of $\overline{\mathcal{S}}$ with respect to $\mathbb{G}$ is

$$
\begin{aligned}
\delta^{2} \mathcal{A}_{\mathbb{G}}(\overline{\mathcal{S}})(\mathbb{J} \nabla u) & =\epsilon \int_{\overline{\mathcal{S}}}\left(\left(\Delta_{g} u\right)^{2}-\operatorname{Ric}^{\mathbb{G}}(\nabla u, \nabla u)\right) \\
& =\epsilon \int_{\mathbb{S}_{\epsilon_{1}}^{1} \times \mathbb{S}_{\epsilon_{4}}^{1}}\left(\left(\epsilon_{3} u_{s s}+\epsilon_{2} u_{t t}\right)^{2}-2\left(\epsilon_{1} u_{s}^{2}+\epsilon_{4} u_{t}^{2}\right)\right) d s d t,
\end{aligned}
$$

while the Hamiltonian second variation of the area of $\overline{\mathcal{S}}$ with respect to $\mathbb{G}^{\prime}$ is

$$
\begin{aligned}
\delta^{2} \mathcal{A}_{\mathbb{G}^{\prime}}(\overline{\mathcal{S}})\left(\mathbb{J}^{\prime} \nabla u\right) & =\epsilon^{\prime} \int_{\overline{\mathcal{S}}}\left(\left(\Delta_{g^{\prime}} u\right)^{2}-\operatorname{Ric}^{\mathbb{G}^{\prime}}(\nabla u, \nabla u)\right) \\
& =\epsilon^{\prime} \int_{\mathbb{S}_{\epsilon_{1}}^{1} \times \mathbb{S}_{\epsilon_{4}}^{1}}\left(4 u_{s t}^{2}+2\left(\epsilon_{1} u_{s}^{2}+\epsilon_{4} u_{t}^{2}\right)\right) d s d t
\end{aligned}
$$




\subsubsection{The case of $L\left(\mathbb{S}^{3}\right)$}

In this case we have $\left(\epsilon_{1}, \epsilon_{2}, \epsilon_{3}, \epsilon_{4}\right)=(1,1,1,1)$, so

$$
\delta^{2} \mathcal{A}_{\mathbb{G}}(\overline{\mathcal{S}})(\mathbb{J} \nabla u)=\int_{\mathbb{S}^{1} \times \mathbb{S}^{1}}\left(\left(u_{s s}+u_{t t}\right)^{2}-2\left(u_{s}^{2}+u_{t}^{2}\right)\right) d s d t .
$$

Since $\left(L^{+}\left(\mathbb{S}^{3}\right), \mathbb{J}, \mathbb{G}\right)$ is Kähler-Einstein with scalar curvature 8 and that the first eigenvalue of the Laplacian on $\mathbb{S}^{1} \times \mathbb{S}^{1}$ endowed with the flat metric is 1 , we deduce that that $\overline{\mathcal{S}}$ is H-unstable with respect to $\mathbb{G}$. One could also make a direct proof, getting both a positive and a negative second variation considering $u_{1}=\cos (s+t)$ and $u_{2}=\cos (2 s)$, so the second variation is indefinite ${ }^{4}$. On the other hand,

$$
\delta^{2} \mathcal{A}_{\mathbb{G}^{\prime}}(\overline{\mathcal{S}})\left(\mathbb{J}^{\prime} \nabla u\right)=\int_{\mathbb{S}^{1} \times \mathbb{S}^{1}}\left(4 u_{s t}^{2}+2\left(u_{s}^{2}+u_{t}^{2}\right)\right) d s d t,
$$

which is positive.

\subsubsection{The case of an unbounded geodesic of $L\left(d \mathbb{S}^{3}\right)$}

Here the metric is $\langle., .\rangle_{1}:=-d x_{1}^{2}+d x_{2}^{2}+d x_{3}^{2}+d x_{4}^{2}$. Hence an unbounded geodesic is, modulo congruence, $\gamma(s)=(\sinh s, \cosh s, 0,0)$, so $\gamma^{\prime}=(\cosh s, \sinh s, 0,0)$ and $\epsilon_{1}=-1$. Hence we may choose for the normal vectors $n_{1}=(0,0,1,0)$ and $n_{2}=(0,0,0,1)$, which implies $\epsilon=1$ and $\epsilon_{2}=1$. Hence $\epsilon^{\prime}=-1$ and $\epsilon_{4}=1$. Finally $\left(\epsilon_{1}, \epsilon_{2}, \epsilon_{3}, \epsilon_{4}\right)=(-1,1,-1,1)$, so we get

$$
\delta^{2} \mathcal{A}_{\mathbb{G}}(\overline{\mathcal{S}})(\mathbb{J} \nabla u)=\int_{\mathbb{R} \times \mathbb{S}^{1}}\left(\left(-u_{s s}+u_{t t}\right)^{2}+2 u_{s}^{2}-2 u_{t}^{2}\right) d s d t .
$$

We claim that the latter is indefinite: on the one hand, we have $\delta^{2} \mathcal{A}_{\mathbb{G}}(\overline{\mathcal{S}})(\mathbb{J} \nabla a(s))=2 \pi \int_{\mathbb{R}}\left(\left(a^{\prime \prime}\right)^{2}+\right.$ $\left.2\left(a^{\prime}\right)^{2}\right) d s>0$ and on the other hand $\delta^{2} \mathcal{A}_{\mathbb{G}}(\overline{\mathcal{S}})(\mathbb{J} \nabla(a(s) \cos t))=\pi \int_{\mathbb{R}}\left(\left(a^{\prime \prime}\right)^{2}+4\left(a^{\prime}\right)^{2}-a^{2}\right) d s$ which may take negative values.

Analogously, we obtain

$$
\delta^{2} \mathcal{A}_{\mathbb{G}^{\prime}}(\overline{\mathcal{S}})\left(\mathbb{J}^{\prime} \nabla u\right)=-\int_{\mathbb{R} \times \mathbb{S}^{1}}\left(4 u_{s t}^{2}-2 u_{s}^{2}+u_{t}^{2}\right) d s d t,
$$

which is indefinite as well, since, for example $\delta^{2} \mathcal{A}_{\mathbb{G}^{\prime}}(\overline{\mathcal{S}})\left(\mathbb{J}^{\prime} \nabla a(s)\right)=4 \pi \int_{\mathbb{R}}\left(a^{\prime}\right)^{2} d s>0$ and $\delta^{2} \mathcal{A}_{\mathbb{G}^{\prime}}(\overline{\mathcal{S}})\left(\mathbb{J}^{\prime} \nabla(a(s) \cos t)\right)=-\pi \int_{\mathbb{R}}\left(2\left(a^{\prime}\right)^{2}+a^{2}\right) d s<0$.

\subsubsection{The case of a definite tube over a closed geodesic of $L\left(d \mathbb{S}^{3}\right)$}

A closed geodesic is, modulo congruence, $\gamma(s)=(0,0, \cos s, \sin s)$, so $\gamma^{\prime}=(0,0,-\sin s, \cos s)$ and $\epsilon_{1}=1$. In order to get a tube with definite metric, we choose $n_{1}=(1,0,0,0)$ and $n_{2}=(0,1,0,0)$, which yields $\epsilon=-1$ and $\epsilon_{2}=1$. Hence $\epsilon^{\prime}=1$ and $\epsilon_{4}=-1$. Finally $\left(\epsilon_{1}, \epsilon_{2}, \epsilon_{3}, \epsilon_{4}\right)=(1,1,-1,-1)$, so we get

$$
\delta^{2} \mathcal{A}_{\mathbb{G}}(\overline{\mathcal{S}})(\mathbb{J} \nabla u)=-\int_{\mathbb{S}^{1} \times \mathbb{R}}\left(\left(-u_{s s}+u_{t t}\right)^{2}-2 u_{s}^{2}+2 u_{t}^{2}\right) d s d t,
$$

\footnotetext{
${ }^{4}$ The H-instability of $\overline{\mathcal{S}}$ with respect to $\mathbb{G}$ was proved in $[\mathrm{Pa}]$.
} 
and

$$
\delta^{2} \mathcal{A}_{\mathbb{G}^{\prime}}(\overline{\mathcal{S}})\left(\mathbb{J}^{\prime} \nabla u\right)=\int_{\mathbb{S}^{1} \times \mathbb{R}}\left(4 u_{s t}^{2}+2 u_{s}^{2}-2 u_{t}^{2}\right) d s d t,
$$

which are both indefinite, as in the previous subsection.

\subsubsection{The case of an indefinite tube over a closed geodesic of $L\left(d \mathbb{S}^{3}\right)$}

Again we have $\gamma(s)=(0,0, \cos s, \sin s)$, so $\gamma^{\prime}=(0,0,-\sin s, \cos s)$ and $\epsilon_{1}=1$. In order to get a tube with indefinite metric, we choose $n_{1}=(0,1,0,0)$ and $n_{2}=(1,0,0,0)$, which yields $\epsilon=1$ and $\epsilon_{2}=-1$. Hence $\epsilon^{\prime}=-1$ and $\epsilon_{4}=-1$. It follows that

$$
\delta^{2} \mathcal{A}_{\mathbb{G}}(\overline{\mathcal{S}})(\mathbb{J} \nabla u)=\int_{\mathbb{S}^{1} \times \mathbb{R}}\left(\left(u_{s s}-u_{t t}\right)^{2}-2 u_{s}^{2}+2 u_{t}^{2}\right) d s d t
$$

and

$$
\delta^{2} \mathcal{A}_{\mathbb{G}^{\prime}}^{\prime}(\overline{\mathcal{S}})\left(\mathbb{J}^{\prime} \nabla u\right)=-\int_{\mathbb{S}^{1} \times \mathbb{R}}\left(4 u_{s t}^{2}+2 u_{s}^{2}-2 u_{t}^{2}\right) d s d t .
$$

As above, both quadratic forms are indefinite.

\subsubsection{The case of a closed geodesic of $L\left(A d \mathbb{S}^{3}\right)$}

In the same way as above, we obtain

$$
\delta^{2} \mathcal{A}_{\mathbb{G}}(\overline{\mathcal{S}})(\mathbb{J} \nabla u)=-\int_{\mathbb{S}^{1} \times \mathbb{S}^{1}}\left(\left(u_{s s}+u_{t t}\right)^{2}-2 u_{s}^{2}-2 u_{t}^{2}\right) d s d t,
$$

which is indefinite (exactly as in the spherical case), and

$$
\delta^{2} \mathcal{A}_{\mathbb{G}^{\prime}}(\overline{\mathcal{S}})\left(\mathbb{J}^{\prime} \nabla u\right)=-\int_{\mathbb{S}^{1} \times \mathbb{S}^{1}}\left(4 u_{s t}^{2}+2\left(u_{s}^{2}+u_{t}^{2}\right)\right) d s d t
$$

which is negative.

\subsubsection{The case of an indefinite tube over an unbounded closed geodesic of $L\left(A d \mathbb{S}^{3}\right)$}

Proceeding as in the previous sections, we obtain

$$
\delta^{2} \mathcal{A}_{\mathbb{G}}(\overline{\mathcal{S}})(\mathbb{J} \nabla u)=-\int_{\mathbb{R}^{2}}\left(\left(u_{s s}+u_{t t}\right)^{2}+2\left(u_{s}^{2}+u_{t}^{2}\right)\right) d s d t,
$$

which is clearly negative, and

$$
\delta^{2} \mathcal{A}_{\mathbb{G}^{\prime}}(\overline{\mathcal{S}})\left(\mathbb{J}^{\prime} \nabla u\right)=-\int_{\mathbb{R}^{2}}\left(4 u_{s t}^{2}-2\left(u_{s}^{2}+u_{t}^{2}\right)\right) d s d t
$$

which is indefinite, by an argument similar to that of Lemma 6 of Section 3.3: setting $u^{\lambda}(s, t):=$ $u(\lambda s, \lambda t)$, we have

$$
\delta^{2} \mathcal{A}_{\mathbb{G}^{\prime}}(\overline{\mathcal{S}})\left(\mathbb{J}^{\prime} \nabla u^{\lambda}\right)=-4 \lambda^{2} \int_{\mathbb{R}^{2}} u_{s t}^{2} d s d t+2 \int_{\mathbb{R}^{2}}\left(u_{s}^{2}+u_{t}^{2}\right) d s d t .
$$




\subsubsection{The case of a definite tube over an unbounded closed geodesic of $L\left(A d \mathbb{S}^{3}\right)$}

Here we have

$$
\delta^{2} \mathcal{A}_{\mathbb{G}}(\overline{\mathcal{S}})(\mathbb{J} \nabla u)=\int_{\mathbb{R}^{2}}\left(\left(u_{s s}+u_{t t}\right)^{2}+2\left(u_{s}^{2}+u_{t}^{2}\right)\right) d s d t
$$

which is positive,

$$
\delta^{2} \mathcal{A}_{\mathbb{G}^{\prime}}(\overline{\mathcal{S}})\left(\mathbb{J}^{\prime} \nabla u\right)=\int_{\mathbb{R}^{2}}\left(4 u_{s t}^{2}-2\left(u_{s}^{2}+u_{t}^{2}\right)\right) d s d t,
$$

which is indefinite as it has been seen in the previous subsection.

\subsubsection{The case of $L\left(\mathbb{H}^{3}\right)$}

We have

$$
\delta^{2} \mathcal{A}_{\mathbb{G}}(\overline{\mathcal{S}})(\mathbb{J} \nabla u)=-\int_{\mathbb{R} \times \mathbb{S}^{1}}\left(\left(u_{s s}-u_{t t}\right)^{2}+2 u_{s}^{2}-2 u_{t}^{2}\right) d s d t,
$$

which is indefinite (see Section 3.4.2.) and

$$
\left.\delta^{2} \mathcal{A}_{\mathbb{G}^{\prime}}(\overline{\mathcal{S}})\left(\mathbb{J}^{\prime} \nabla u\right)=\int_{\mathbb{R} \times \mathbb{S}^{1}}\left(4 u_{s t}^{2}-2 u_{s}^{2}+2 u_{t}^{2}\right)\right) d s d t,
$$

which is indefinite as well.

\begin{tabular}{|l||l||c|c|c|c|c|}
\hline Space form & Type of $\gamma$ & Induced metric on $\mathcal{S}$ & $\left(\epsilon_{1}, \epsilon_{2}, \epsilon_{3}, \epsilon_{4}\right)$ & Topology of $\mathcal{S}$ & $\mathbb{G}$-H-stability & $\mathbb{G}^{\prime}$-H-stability \\
\hline \hline $\mathbb{S}_{0}^{3}=\mathbb{S}^{3}$ & closed & definite & $(1,1,1,1)$ & torus & unstable & stable \\
\hline $\mathbb{S}_{1}^{3}=d \mathbb{S}^{3}$ & closed & definite & $(1,1,-1,-1)$ & cylinder & unstable & unstable \\
\hline & closed & indefinite & $(1,-1,1,-1)$ & cylinder & unstable & unstable \\
\hline & unbounded & indefinite & $(-1,1,-1,1)$ & cylinder & unstable & unstable \\
\hline $\mathbb{S}_{2}^{3}=A d \mathbb{S}^{3}$ & closed & indefinite & $(1,-1,-1,1)$ & torus & unstable & stable \\
\hline & unbounded & indefinite & $(-1,1,1,-1)$ & plane & stable & unstable \\
\hline & unbounded & definite & $(-1,-1,-1,-1)$ & plane & stable & unstable \\
\hline $\mathbb{S}_{3}^{3}=\mathbb{H}^{3}$ & unbounded & definite & $(-1,-1,1,1)$ & cylinder & unstable & unstable \\
\hline
\end{tabular}

\subsection{Rank 1 Lagrangian surfaces in the tangent bundle of a Riemann surface}

Let $\left(\mathcal{N}, g_{0}\right)$ be an oriented Riemannian surface with metric $g_{0}$ and denote by $j$ the complex structure induced from its orientation (i.e. the rotation of angle $\pi / 2$ ). It has been proved in [GK] (cf also $[$ AGR],[An2]) that the tangent bundle of $\mathcal{N}$ enjoys a natural pseudo-Kähler structure $(\mathbb{J}, \mathbb{G})$, which may be described as follows: the Levi-Civita connection of $g$ induces a splitting of $T T \mathcal{N}$ into

$$
\begin{aligned}
T T \mathcal{N}=H \mathcal{N} \oplus V \mathcal{N} & \simeq T \mathcal{N} \oplus T \mathcal{N} \\
X & \simeq\left(X^{h}, X^{v}\right),
\end{aligned}
$$


According to this decomposition, we set

$$
\begin{aligned}
\mathbb{J}\left(X^{h}, X^{v}\right) & :=\left(j X^{h}, j X^{v}\right) \\
\mathbb{G}\left(\left(X^{h}, X^{v}\right),\left(Y^{h}, Y^{v}\right)\right) & :=g_{0}\left(X^{v}, j Y^{h}\right)-g_{0}\left(X^{h}, j Y^{v}\right) .
\end{aligned}
$$

It has been proved in $[\mathrm{GK}]$ (see also $[\mathrm{AR}]$ ) that $(T \mathcal{N}, \mathbb{J}, \mathbb{G}$ ) is a pseudo-Kähler manifold and that $\mathbb{G}$ have neutral signature. The next proposition describes a class of $\mathrm{H}$-minimal Lagrangian surfaces of $(T \mathcal{N}, \mathbb{J}, \mathbb{G})$ :

Proposition 3 ([AGR]) Let $\gamma: I \rightarrow \mathcal{N}$ be a regular curve parametrized by arclength and a real map a $\in C^{1}(I)$. Then the surface $\mathcal{L}$ image of the immersion

$$
\begin{aligned}
f: I \times \mathbb{R} & \rightarrow T \mathcal{N} \\
(s, t) & \mapsto\left(\gamma(s), a(s) \gamma^{\prime}(s)+t j \gamma^{\prime}(s)\right),
\end{aligned}
$$

is a H-minimal, flat, Lagrangian surface. Moreover, it is minimal if and only if $\gamma$ is a geodesic. These surfaces are characterized by the fact that the restriction to $\mathcal{L}$ of the canonical projection $\pi: T \mathcal{N} \rightarrow \mathcal{N}$ has rank one.

In order to study the H-stability of such surfaces (Theorem 5), we shall need the following description of the Ricci curvature of the metric $\mathbb{G}$ :

Proposition 4 ([AR]) Let $X$ and $Y$ and two vector fields on $T \mathcal{N}$ and $K$ the Gaussian curvature of $\left(\mathcal{N}, g_{0}\right)$. Then the Ricci curvature of $(T \mathcal{N}, \mathbb{G})$ is given by

$$
\operatorname{Ric}^{\mathbb{G}}(X, Y)=2 \operatorname{Ric}^{g_{0}}\left(X^{h}, Y^{h}\right)=2 K g_{0}\left(X^{h}, Y^{h}\right) .
$$

Proof of the Theorem 5: An easy calculation (cf $[\mathrm{AGR}]$ ) shows that the coefficients of the induced metric $f^{*} \mathbb{G}$ in the coordinates $(s, t)$ are

$$
\left(\begin{array}{cc}
-2 a \kappa & -1 \\
-1 & 0
\end{array}\right)
$$

If follows that the gradient of the induced metric takes the following form

$$
\nabla u=-u_{t} \partial_{s}+\left(-u_{s}+2 a \kappa u_{t}\right) \partial_{t} .
$$

On the other hand, the coefficients of the second fundamental form $h$ are

$$
h_{112}=\kappa \quad h_{122}=0 \quad \text { and } \quad h_{222}=0,
$$

so in particular the mean curvature vector is $2 \vec{H}=\kappa \rrbracket \partial_{t}$. Hence

$$
\mathbb{G}(2 \vec{H}, \mathbb{J} \nabla u)=-\mathbb{G}(\mathbb{J} 2 \vec{H}, \nabla u)=-\mathbb{G}\left(-\kappa \partial_{t},-u_{t} \partial_{s}+\left(-u_{s}+2 a \kappa u_{t}\right) \partial_{t}\right)=-\mathbb{G}\left(\kappa \partial_{t}, u_{t} \partial_{s}\right)=\kappa u_{t} .
$$

Next we have

$$
h(\nabla u, \nabla u)=u_{t}^{2} h\left(\partial_{s}, \partial_{s}\right)-2 u_{t}\left(2 a \kappa u_{t}-u_{s}\right) h\left(\partial_{t}, \partial_{s}\right)+\left(2 a \kappa u_{t}-u_{s}\right)^{2} h\left(\partial_{t}, \partial_{t}\right),
$$


SO

$$
\begin{aligned}
\mathbb{G}(h(\nabla u, \nabla u), 2 \vec{H}) & =\kappa \mathbb{G}\left(h(\nabla u, \nabla u), \mathbb{J} \partial_{t}\right) \\
& =\kappa\left(u_{t}^{2} h_{112}-2 u_{t}\left(-u_{s}+2 a \kappa u_{t}\right) h_{112}+\left(-u_{s}+2 a \kappa u_{t}\right)^{2} h_{222}\right) \\
& =\kappa^{2} u_{t}^{2} .
\end{aligned}
$$

By Proposition 4 we have

$$
\operatorname{Ric}^{\mathbb{G}}\left(\partial_{s}, \partial_{s}\right)=2 K \quad \operatorname{Ric}^{\mathbb{G}}\left(\partial_{s}, \partial_{t}\right)=\operatorname{Ric}^{\mathbb{G}}\left(\partial_{t}, \partial_{t}\right)=0,
$$

so

$$
\operatorname{Ric}^{\mathbb{G}}(\nabla u, \nabla u)=2 K u_{t}^{2} .
$$

By the Main Theorem, we obtain

$$
\delta^{2} \mathcal{A}(\mathcal{L})(\mathbb{\mathbb { J }} \nabla u)=\int_{I \times \mathbb{R}}\left(4 u_{s t}^{2}-\left(\kappa^{2}+2 K\right) u_{t}^{2}\right) d s d t .
$$

In particular, if $\kappa^{2} \leq-2 K$ along the curve $\gamma$, the Hamiltonian second variation is obviously positive.

We now claim that if $\gamma$ is unbounded and $\kappa^{2}>-2 K$, then $\mathcal{L}$ is H-unstable. To see this, set $u^{\lambda}(s, t):=\lambda^{3 / 2} u(\lambda s, t)$, so that

$$
\delta^{2} \mathcal{A}(\mathcal{L})\left(\mathbb{J} \nabla u^{\lambda}\right)=\lambda^{2} \int_{\mathbb{R}^{2}} 4 u_{s t}^{2} d s d t-\int_{\mathbb{R}^{2}}\left(\kappa^{2}\left(\lambda^{-1} s\right)+2 K\left(\lambda^{-1} s\right)\right) u_{t}^{2} d s d t,
$$

so letting $\lambda$ tend to 0 and $\infty$ respectively yields positive and negative variations.

Finally, we assume that $\gamma$ is a closed curve of length $L$. Then, using Wirtinger's inequality for $L$-periodic functions, we have

$$
\begin{aligned}
\delta^{2} \mathcal{A}(\mathcal{L})(\mathbb{d} \nabla u) & =\int_{\mathbb{R} / L \mathbb{Z} \times \mathbb{R}}\left(4 u_{s t}^{2}-\left(\kappa^{2}+2 K\right) u_{t}^{2}\right) d s d t \\
& \geq \frac{4 \pi^{2}}{L^{2}} \int_{\mathbb{R} / L \mathbb{Z} \times \mathbb{R}} 4 u_{t}^{2} d s d t-\left(\sup _{\mathbb{R} / L \mathbb{Z}}\left(\kappa^{2}+2 K\right)\right) \int_{\mathbb{R} / L \mathbb{Z} \times \mathbb{R}} u_{t}^{2} d s d t \\
& \geq\left(\frac{16 \pi^{2}}{L^{2}}-\sup _{\mathbb{R} / L \mathbb{Z}}\left(\kappa^{2}+2 K\right)\right) \int_{\mathbb{R} / L \mathbb{Z} \times \mathbb{R}} u_{t}^{2} d s d t .
\end{aligned}
$$

Hence, if $\sup _{\gamma}\left(\kappa^{2}+2 K\right) \leq \frac{16 \pi^{2}}{L^{2}}$, then $\mathcal{L}$ is H-stable.

\section{Appendix}

The purpose of this Appendix is the proof of the following:

Pseudo Riemannian Bochner's formula Let $(\mathcal{L}, g)$ be a pseudo-Riemannian manifold with Ricci tensor Ric ${ }^{\mathcal{L}}$ and $u$ a smooth, compactly supported function on $\mathcal{L}$. Then

$$
\frac{1}{2} \Delta(g(\nabla u, \nabla u))=\operatorname{Ric}^{\mathcal{L}}(\nabla u, \nabla u)+g(\nabla u, \nabla(\Delta u))+g\left(\nabla^{2} u, \nabla^{2} u\right) .
$$




\section{Proof.}

Let $\left(e_{1}, \ldots, e_{n}\right)$ be a local orthonormal frame which is normal at some point $x$, i.e. $\nabla_{e_{i}} e_{j}(x)$ vanishes. This implies that $\nabla_{\nabla u} e_{i}=\sum \epsilon_{i} e_{i}(u) \nabla_{e_{i}} e_{j}(x)$ vanishes as well. We set as usual $\epsilon_{i}:=g\left(e_{i}, e_{i}\right)$. Then we have

$$
\begin{aligned}
\frac{1}{2} \Delta g(\nabla u, \nabla u) & =\frac{1}{2} \sum_{i=1}^{n} \epsilon_{i} e_{i}\left(e_{i}(g(\nabla u, \nabla u))\right) \\
& =\sum_{i=1}^{n} \epsilon_{i} e_{i}\left(g\left(\nabla_{e_{i}} \nabla u, \nabla u\right)\right) \\
& =\sum_{i=1}^{n} \epsilon_{i} e_{i}\left(\nabla^{2} u\left(e_{i}, \nabla u\right)\right) \\
& \left.=\sum_{i=1}^{n} \epsilon_{i} e_{i}\left(g\left(\nabla_{\nabla u} \nabla u, e_{i}\right)\right)\right) \\
& \left.=\sum_{i=1}^{n} \epsilon_{i} g\left(\nabla_{e_{i}} \nabla_{\nabla u} \nabla u, e_{i}\right)\right) \\
& \left.=\sum_{i=1}^{n} \epsilon_{i}\left(g\left(R\left(\nabla u, e_{i}\right) \nabla u, e_{i}\right)\right)+g\left(\nabla_{\nabla u} \nabla_{e_{i}} \nabla u, e_{i}\right)-g\left(\nabla_{\left[\nabla u, e_{i}\right]} \nabla u, e_{i}\right)\right) \\
& =R i c^{\mathcal{L}}(\nabla u, \nabla u)+\sum_{i=1}^{n} \epsilon_{i}\left(g\left(\nabla_{\nabla u} \nabla_{e_{i}} \nabla u, e_{i}\right)-g\left(\nabla_{\left[\nabla u, e_{i}\right]} \nabla u, e_{i}\right)\right)
\end{aligned}
$$

We now deal with the first term of the sum on the right hand side of the last expression:

$$
\begin{aligned}
\sum_{i=1}^{n} \epsilon_{i} g\left(\nabla_{\nabla u} \nabla_{e_{i}} \nabla u, e_{i}\right) & =\sum_{i=1}^{n} \epsilon_{i}\left((\nabla u) g\left(\nabla_{e_{i}} \nabla u, e_{i}\right)-g\left(\nabla_{e_{i}} \nabla u, \nabla_{\nabla u} e_{i}\right)\right) \\
& =\left(\sum_{i=1}^{n} \epsilon_{i}\left((\nabla u) g\left(\nabla_{e_{i}} \nabla u, e_{i}\right)\right)-0\right. \\
& =\nabla u\left(\sum_{i=1}^{n} \epsilon_{i} g\left(\nabla_{e_{i}} \nabla u, e_{i}\right)\right) \\
& =\nabla u(\operatorname{div} \nabla u) \\
& =\nabla u(\Delta u) \\
& =g(\nabla u, \nabla(\Delta u)) .
\end{aligned}
$$

Finally, we have, recalling that $\nabla_{\nabla u} e_{i}(x)$ vanishes,

$$
\begin{aligned}
& \sum_{i=1}^{n} \epsilon_{i} g\left(\nabla\left[\nabla u, e_{i}\right] \nabla u, e_{i}\right)=\sum_{i=1}^{n} \epsilon_{i} \nabla^{2} u\left(\left[\nabla u, e_{i}\right], e_{i}\right) \\
& =\sum_{i=1}^{n} \epsilon_{i} \nabla^{2} u\left(\nabla_{\nabla u} e_{i}-\nabla_{e_{i}} \nabla u, e_{i}\right)
\end{aligned}
$$




$$
\begin{aligned}
& =-\sum_{i=1}^{n} \epsilon_{i} \nabla^{2} u\left(\nabla_{e_{i}} \nabla u, e_{i}\right) \\
& =-g\left(\nabla^{2} u, \nabla^{2} u\right) .
\end{aligned}
$$

Finally, we get

$$
\frac{1}{2} \Delta(g(\nabla u, \nabla u))=\operatorname{Ric}^{\mathcal{L}}(\nabla u, \nabla u)+g(\nabla u, \nabla(\Delta u))+g\left(\nabla^{2} u, \nabla^{2} u\right),
$$

the required formula.

\section{References}

[AGK] D. Alekseevsky, B. Guilfoyle, W. Klingenberg, On the geometry of spaces of oriented geodesics, Ann. Global Anal. Geom. 40 (2011) 1-21

[An1] H. Anciaux, An isoperimetric inequality for Hamiltonian stationary Lagrangian tori in $\mathbb{C}^{2}$ related to Oh's conjecture, Math. Zeitschrift 241 (2002) 639-664

[An2] H. Anciaux, Minimal submanifolds in pseudo-Riemannian geometry, World Scientific, (2010)

[An3] H. Anciaux, Minimal Lagrangian submanifolds in indefinite complex space, arXiv:1011.3756

[An4] H. Anciaux, Space of geodesics of pseudo-Riemannian space forms and normal congruences of hypersurfaces, arXiv:1112.1758

[AGR] H. Anciaux, B. Guilfoyle, P. Romon, Minimal submanifolds in the tangent bundle of a Riemannian surface, J. Geometry and Physics 61 (2011) 237-247

[AR] H. Anciaux, P. Romon, A natural structure on the tangent bundle of a pseudo- or para-Kähler manifold, in preparation

[Dm] P. Dombrowski, On the geometry of the tangent bundle, J. Reine Angew. Math. 210 (1962) 73-88

[Dg] Y. Dong, On Indefinite Special Lagrangian Submanifolds in Indefinite Complex Euclidean Spaces, J. of Geom. and Physics 59 (2009) 710-726

[Ha] M. Haskins, The geometry complexity of special Lagrangian $T^{2}$-cones, Invent. Math. 157 (2004) 11-70

[GK] B. Guilfoyle, W. Klingenberg, Area-stationary surfaces in certain neutral Kähler 4manifolds, Beiträge Algebra Geom. 49 (2008) no. 2, 481-490

[GG1] N. Georgiou, B. Guilfoyle, On the space of oriented geodesics of hyperbolic 3-space, Rocky Mountain J. Math. 40 (2010) 1183-1219 
[GG2] N. Georgiou, B. Guilfoyle, A characterization of Weingarten surfaces in hyperbolic space, Abh. Math. Sem. Hamburg 80 (2010) 233-253

[Ge] N. Georgiou, On area stationary surfaces in the space of oriented geodesics of hyperbolic 3-space, to appear in Math. Scand.

[HL1] R. Harvey, H.B. Lawson, Calibrated geometries, Acta Math. 148 (1982) 47-157

[HL2] R. Harvey, H.B. Lawson, Split Special Lagrangian Geometry, arXiv:1007.0450

[Me] J. Mealy, Volume maximization in Semi-Riemannian Manifolds, Indiana Univ. Math. J. 40 (1991) 793-814

[NN] A. Newlander, L. Nirenberg, Complex analytic coordinates in almost complex manifolds, Annals of Math. (2) 65 (1957) 391-404

[Oh1] Y.G. Oh, Second variation and stabilities of minimal lagrangian submanifolds in Kähler manifolds, Invent. Math. 101 (1990) 501-519

[Oh2] Y.G. Oh, Volume minimization of Lagrangian submanifolds under Hamiltonian deformations, Math. Z. 212 (1993) 175-192

[Pa] B. Palmer, Hamiltonian minimality and Hamiltonian stability of Gauss maps, Diff. Geom. Appl. 7 (1997) no. 1, 51-58

[Re] R. Reilly, Applications of the Hessian operator in a Riemannian manifold, Indiana Univ. Math. J. 26 (1977) 459-472

[Sa] M. Salvai, On the geometry of the space of oriented lines of hyperbolic space, Glasg. Math. J. 49 (2007) 357-366

[Si] J. Simons, Minimal varieties in Riemannian manifolds Ann. Math. 88 (1968) 82-105

[Ur1] F. Urbano, Index of Lagrangian submanifolds of $\mathbb{C P}^{n}$ and the Laplacian 1-forms, Indiana Univ. Math. J. 48 (1993) 309-318

[Ur2] F. Urbano, Hamiltonian stability and index of minimal Lagrangian surfaces in complex projective plane, Indiana Univ. Math. J. 56 (2007) 931-946

[Xi] Y. Xin, Minimal submanifolds and related topics, Nankai tracts in mathematics, Vol. 8 (2001), World Scientific

Henri Anciaux

Universidade de São Paulo, IME

1010 Rua do Matão, Cidade Universitária 05508-090 São Paulo, Brazil

henri.anciaux@gmail.com
Nikos Georgiou

Department of Mathematics and Statistics

University of Cyprus

1678 Nicosia, Cyprus

georgiou.g.nicos@ucy.ac.cy 